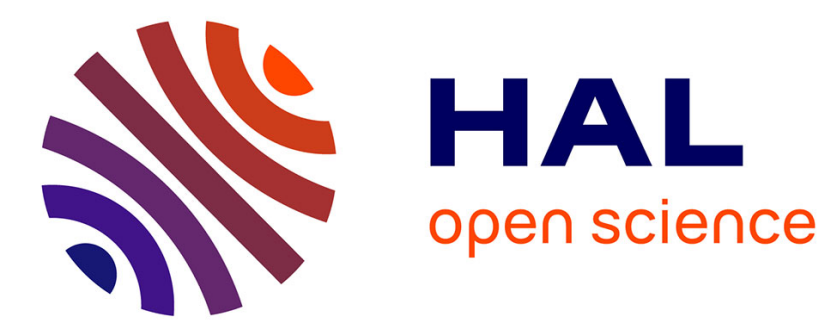

\title{
Post-buckling of an elastic plate over an inviscid and buoyant fluid
}

Yves Leroy, Martin Guiton, Nicolas Triantafyllidis

\section{To cite this version:}

Yves Leroy, Martin Guiton, Nicolas Triantafyllidis. Post-buckling of an elastic plate over an inviscid and buoyant fluid. International Journal of Solids and Structures, 2002, 39, pp.3873-3891. 10.1016/S0020-7683(02)00185-3 . hal-00111354

\section{HAL Id: hal-00111354 https://hal.science/hal-00111354}

Submitted on 28 Jun 2019

HAL is a multi-disciplinary open access archive for the deposit and dissemination of scientific research documents, whether they are published or not. The documents may come from teaching and research institutions in France or abroad, or from public or private research centers.
L'archive ouverte pluridisciplinaire HAL, est destinée au dépôt et à la diffusion de documents scientifiques de niveau recherche, publiés ou non, émanant des établissements d'enseignement et de recherche français ou étrangers, des laboratoires publics ou privés. 


\title{
Post-buckling of an elastic plate over an inviscid and buoyant fluid
}

\author{
Yves M. Leroy ${ }^{\mathrm{a}, *}$, Martin L.E. Guiton ${ }^{\mathrm{a}}$, Nicolas Triantafyllidis ${ }^{\mathrm{b}}$ \\ a Laboratoire de Mécanique des Solides, U.M.R. C.N.R.S. no. 7649, Ecole Polytechnique, 91128 Palaiseau Cedex, France \\ ${ }^{\mathrm{b}}$ Department of Aerospace Engineering, The University of Michigan, Ann Arbor, MI 48109-2140, USA
}

The model problem proposed to study the buckling of stratified geological layers consists of a linearly elastic plate, capable of accommodating finite rotations, resting over an inviscid and buoyant fluid. The Lyapunov-Schmidt-Koiter decomposition is applied to construct the bifurcated equilibrium solutions. The asymptotic analysis of the postbuckling reveals a decrease in the magnitude of the lateral compressive force during an overall shortening of the stratified structure. Buckling and post-buckling are not influenced by the presence of a vertical stress gradient in the elastic plate.

\section{Introduction}

The layered structure considered here is a model problem often encountered in geological applications with length scales ranging from sedimentary basins (Triantafyllidis and Leroy, 1997) to the lithospheric thickness (McAdoo and Sandwell, 1985; Martinod and Davy, 1992; Wallace and Melosh, 1994).

The rock rheology during buckling departs from linear elasticity, an hypothesis considered here, because the stress prevailing at the onset of buckling is sufficient to trigger irreversible deformation mechanisms (Ramberg and Stephansson, 1964). However, the elastic stiffness of the structure can still control the initial buckling of slender plates as well as the initial buckling mode development if the permanent deformation is not generalized to the whole structure, as it was shown in the case of the buckling of a plate with diffuse fracturing by Guiton et al. (2001). Also, an elastic unloading during buckling of an elasto-plastic plate could lead to the arrest of the fold development in an elastic equilibrium state (Massin et al., 1996). It is for these reasons that the complete post-buckling analysis of the conservative system composed of an elastic plate over a buoyant inviscid fluid, not found in the literature, is a prerequisite to progress in our understanding of fold development.

The paper contents are as follows. Section 2 presents the model problem as well as the construction of the total energy of the structure under initial stress. The selection of the appropriate strain measure for a

\footnotetext{
* Corresponding author. Fax: +33-1-69-33-30-26.

E-mail address: leroyy@1ms.polytechnique.fr (Y.M. Leroy).
} 
plate at finite strain is found in Appendix A. Section 3 pertains to the fundamental solution to the problem, characterized by a flat elastic plate, and to the onset of bifurcation. The Lyapunov-Schmidt-Koiter decomposition (which is documented in Budiansky (1974), Potier-Ferry $(1978,1979)$ as well as in Iooss and Joseph (1980)) is then applied to construct the secondary solution corresponding to the development of a fold. The details of the calculations are found in Section 4. Section 5 contains a discussion of the results obtained for a layered structure which is a prototype for a folded section through the Campos basin, Offshore Brazil (Cobbold and Szatmari, 1991). The asymptotic solution is compared to a finite-element approximation which is presented in Appendix B.

\section{Description of the model problem}

The three objectives of this section are to introduce the geometry of the model problem, to define the kinematics of the elastic plate and to set up the energy of the solid-fluid system.

The model problem consists of a plate composed of an elastic isotropic material and having for length $L$ and thickness $H_{\mathrm{s}}$ in the reference configuration, Fig. 1a. This plate is in equilibrium over a buoyant incompressible fluid occupying in the reference configuration a layer of thickness $H_{\mathrm{f}}$. The fluid is assumed inviscid for the time scale of the loading. During loading, the right boundary wall of the layered structure is displaced, while remaining vertical, by either varying the force $P$ or the displacement $\Delta$. The total volume of the fluid being constrained, the fundamental solution to the problem consists of a plate sustaining a homogeneous compression and a vertical translation. One of the objectives of this paper is to derive the loading conditions at which a second solution is possible in the form of a buckle, Fig. 1b. The deformed

(a)

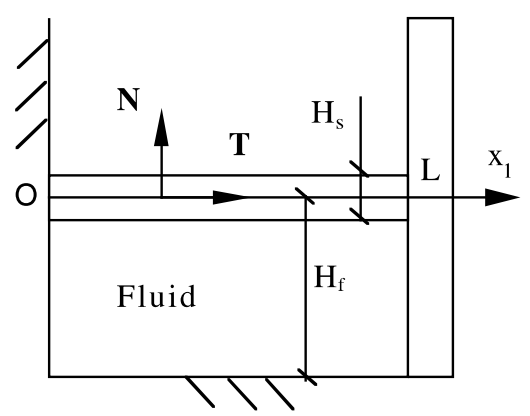

(c)

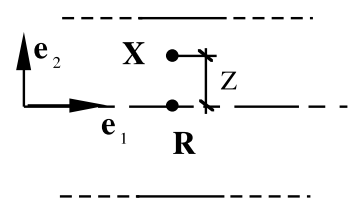

(b) $\mathbf{n}$
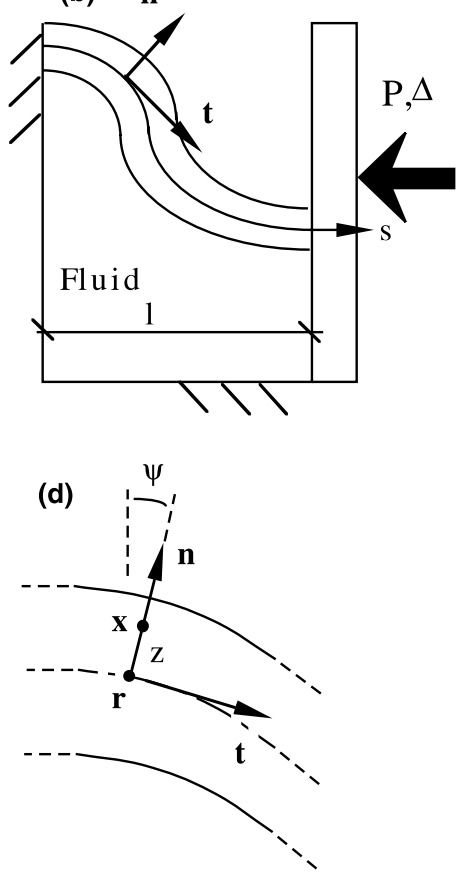

Fig. 1. Geometry of the model problem: an elastic plate on an inviscid fluid in the reference (a) and (c) and in the current (b) and (d) configuration. The variables necessary to define the plate kinematics are defined in (c) and (d). 
plate in either the fundamental mode or the buckled mode shown in that figure corresponds to the current configuration.

The planar kinematics of the deforming plate are now provided. A material point in the plate reference configuration labeled $\mathbf{X}$ is positioned by its projection on the mid-surface, point $\mathbf{R}$, as well as by the distance $Z$ separating the two points, as shown in Fig. 1c. The normal and tangent vectors in the reference configuration mid-surface are noted $\mathbf{N}$ and $\mathbf{T}$ and are the base vectors $\mathbf{e}_{2}$ and $\mathbf{e}_{1}$, respectively. Point $\mathbf{X}$ is transformed to point $\mathbf{x}$ in the current configuration with the assumption that surfaces initially normal to the mid-surface are also perpendicular to the mid-surface in the current configuration, the rotation of the normal being denoted by $\psi$. Consequently, the material point at $\mathbf{X}$ and $\mathbf{R}$ are transformed to $\mathbf{x}$ and $\mathbf{r}$, the latter two points being distant by $z$, Fig. 1d. The normal and tangent vectors in the current configuration are $\mathbf{n}$ and $\mathbf{t}$, respectively. Above and in what follows, bold letters identify vectorial and more generally tensorial quantities.

The studied transformation of the plate is described in two steps. First, the points on the mid-surface are displaced by $v\left(X_{1}\right)$ and $w\left(X_{1}\right)$ in the directions $\mathbf{e}_{1}$ and $\mathbf{e}_{2}$, respectively. These two scalars $v$ and $w$ are function of the first coordinate on the reference configuration $X_{1}$. Note that the boundary condition at $X_{1}=0$ is $v(0)=0$. Second, the displacement $\mathbf{u}$ of point $\mathbf{X}$ is the same as the displacement of its projection $\mathbf{R}$ on the mid-surface plus the difference between the positions along the initial and the current normals

$$
\mathbf{u}\left(X_{1}, Z\right)=v\left(X_{1}\right) \mathbf{T}+w\left(X_{1}\right) \mathbf{N}+(z \mathbf{n}-Z \mathbf{N}) .
$$

It is of interest to express this displacement in terms of the base vectors and for that purpose Serret-Frenet relations in the current configurations are recalled

$$
\begin{aligned}
& \mathbf{t}=\frac{\mathrm{d} \mathbf{r}}{\mathrm{d} s}=\cos \psi \mathbf{e}_{1}+\sin \psi \mathbf{e}_{2} \quad \text { with } \lambda^{2}=\left(1+v_{, 1}\right)^{2}+\left(w_{, 1}\right)^{2}, \quad \cos \psi=\frac{1+v_{, 1}}{\lambda}, \sin \psi=\frac{w_{, 1}}{\lambda}, \\
& \frac{\mathrm{dt}}{\mathrm{d} s}=-\kappa \mathbf{n} \quad \text { with } \kappa\left(X_{1}\right) \equiv-\frac{\psi_{, 1}}{\lambda}=\left(v_{, 11} \sin \psi-w_{, 11} \cos \psi\right) \lambda^{-2},
\end{aligned}
$$

and are complemented by the definition of the curvature $\kappa$, and the stretch ratio $\lambda$ in terms of the displacement. In (2) and in the rest of this paper, a comma means derivative or partial derivative with respect to the variable which follows (e.g. $v_{, 1}$ is $\mathrm{d} v / \mathrm{d} X_{1}$ ). Note again that the angle $\psi$ marks the rotation of the normal with respect to the vertical direction, Fig. 1d, and that the boundary conditions are such that $\psi\left(X_{1}=0\right)=\psi\left(X_{1}=L\right)=0$ for any equilibrium solution. The scalar $s$ in (2) denotes the arc length on the mid-surface of the plate in its current configuration and is a monotonic function of the initial position $X_{1}$. Consequently, any variable defined on the current configuration can also be seen as a function of $X_{1}$. Combining (1) and (2) provides the expression for the displacement in the $\left(\mathbf{e}_{1}, \mathbf{e}_{2}, \mathbf{e}_{3}\right)$ basis

$$
\mathbf{u}\left(X_{1}, Z\right)=\left(v\left(X_{1}\right)-z \sin \psi\right) \mathbf{e}_{1}+\left(w\left(X_{1}\right)+z \cos \psi-Z\right) \mathbf{e}_{2} .
$$

The adopted strain measure which should be based on the gradient of the displacement field in (3) is not the classical Lagrangian strain. It is shown in Appendix A that the appropriate strain measure is

$$
\mathbf{e}=(\epsilon+\beta Z) \mathbf{e}_{1} \otimes \mathbf{e}_{1} \quad \text { with } \epsilon\left(X_{1}\right)=\lambda\left(X_{1}\right)-1 \text { and } \beta\left(X_{1}\right)=\kappa\left(X_{1}\right) \lambda\left(X_{1}\right)
$$

adopting the assumption of plane-strain and disregarding the change in length in the direction normal to the mid-surface $(Z=z)$. This strain tensor has a single component denoted $e$ in the following which is composed of two parts $(e=\epsilon+\beta Z)$. The first, $\epsilon$, is the membrane strain and the second, $\beta Z$, is due to the bending of the plate.

The last objective of this section is to define the total energy of the solid-fluid system. The solid plate is composed of a linear elastic material under initial axial stress described by $\sigma^{\mathrm{p}}(Z)=\sigma_{0}^{\mathrm{p}}+Z k$ in which $\sigma_{0}^{\mathrm{p}}$ and $k$ are the initial stress at mid-surface and the vertical stress gradient, respectively. Integrating the material free energy over the plate thickness results in the following internal energy per unit reference length of the plate 


$$
\phi(\epsilon, \beta)=N^{\mathrm{p}} \epsilon+M^{\mathrm{p}} \beta+\frac{1}{2} E\left(H_{\mathrm{s}} \epsilon^{2}+\beta^{2} I\right) \quad \text { with } N^{\mathrm{p}}=\sigma_{0}^{\mathrm{p}} H_{\mathrm{s}} \text { and } M^{\mathrm{p}}=k I,
$$

in which $E, I, M^{\mathrm{p}}$ and $N^{\mathrm{p}}$ are the modulus of elasticity for a plate $\left(E_{3 \mathrm{D}} /(1-2 v)\right.$ with $v$ denoting Poisson's ratio and $E_{3 \mathrm{D}}$ Young's modulus of elasticity), the plate inertia (short-hand notation for $H_{\mathrm{s}}^{3} / 12$ ) and the initial moment and axial force, respectively. The axial force and the bending moment through any section of the plate are

$$
N\left(X_{1}\right)=N^{\mathrm{p}}+E H_{\mathrm{s}} \epsilon\left(X_{1}\right) \text { and } M\left(X_{1}\right)=M^{\mathrm{p}}+E I \beta\left(X_{1}\right),
$$

and are defined as the partial derivatives of the potential $\phi(\epsilon, \beta)$, defined in (5), with respect to $\epsilon$ and $\beta$, respectively. The total internal energy of the plate $\mathscr{E}_{\mathrm{s}}$ is the integral of the energy density $\phi(\epsilon, \beta)$ over the mid-surface in the reference configuration. The energy of the external forces acting on the plate are due to the gravity field and the action at the right boundary. One needs to integrate this gravity force projected on the displacement vector (3) over the current configuration and to transform the result to the reference configuration to obtain the contribution to the external energy which reads

$$
\mathscr{E}_{\mathrm{s}}^{\mathrm{e}}=-\rho_{\mathrm{s}} H_{\mathrm{s}} \int_{0}^{L} w \mathrm{~d} X_{1}-P_{\mathrm{s}} v(L)
$$

in which $\rho_{\mathrm{s}}$ and $P_{\mathrm{s}}$ are the solid volumetric weight, defined as the solid volumetric mass in the reference configuration times the gravity acceleration, and the force at the boundary acting on the solid plate (positive in compression). Note that the second term in the right-hand side of (7) should only be present for a force control on the boundary.

The fluid is inviscid and incompressible and its contribution to the total energy of the system is computed by taking the difference in potential energy between the current and the reference configurations. In calculating these quantities and for sake of simplicity, the thickness of the fluid layer is taken from the bottom of the fluid layer to the mid-surface of the solid plate, as shown in Fig. 1a and b. This approximation is of course valid as long as the fluid layer thickness is large compared to the plate thickness. The final expression for the energy of the fluid is

$$
\mathscr{E}_{\mathrm{f}}=\rho_{\mathrm{f}} \frac{1}{2}\left(\int_{0}^{L}\left(w+H_{\mathrm{f}}\right)^{2}\left(1+v_{, 1}\right) \mathrm{d} X_{1}-H_{\mathrm{f}}^{2} L\right)+P_{\mathrm{f}} v(L)
$$

in which $\rho_{\mathrm{f}}$ is the fluid volumetric weight, defined as the volumetric mass in the reference configuration times the gravity acceleration. Note again that the last term in the right-hand side of (8) should only be present for a force control.

It should be stressed that the volume occupied by the fluid is constant taking for value $H_{\mathrm{f}} L$. Consequently, the vertical and horizontal displacement of the solid plate are constrained by

$$
\int_{0}^{L}\left(w+H_{\mathrm{f}}\right)\left(1+v_{, 1}\right) \mathrm{d} X_{1}=H_{\mathrm{f}} L .
$$

To account for this constraint, responsible for the overall vertical displacement of the plate during compression, the vertical displacement is decomposed in two parts

$$
w\left(X_{1}\right)=\omega\left(X_{1}\right)+W,
$$

where the scalar $W$ is found from (9) to be

$$
W=\frac{1}{l}\left(-H_{\mathrm{f}} v(L)-\int_{0}^{L} \omega \cos \psi \lambda \mathrm{d} X_{1}\right),
$$

with $l$ standing for the current length $(L+v(L))$ of the structure. 
The total energy of the structure $\mathscr{E}$ is $\mathscr{E}^{\mathrm{i}}-\mathscr{E}^{\mathrm{e}}+_{\mathrm{s}} \mathscr{E}_{\mathrm{f}}$ and its final expression reads

$$
\begin{aligned}
\mathscr{E}= & \int_{0}^{L}\left(N^{\mathrm{p}} \epsilon+M^{\mathrm{p}} \beta+\frac{E}{2}\left(H_{\mathrm{s}} \epsilon^{2}+I \beta^{2}\right)+H_{\mathrm{s}} \rho_{\mathrm{s}} \omega+\frac{1}{2} \rho_{\mathrm{f}} \omega^{2} \lambda \cos \psi\right) \mathrm{d} X_{1}+P v(L)+H_{\mathrm{s}} \rho_{\mathrm{s}} W L \\
& -\rho_{\mathrm{f}} \frac{1}{2}\left(W^{2} l+H_{\mathrm{f}}^{2} v(L)+2 W H_{\mathrm{f}} v(L)\right),
\end{aligned}
$$

which is obtained by adding the integral over the plate length of (5)-(8), subtracting (7), introducing the decomposition (10) and the constraint expressed in (9) and through the definition of $W$ in (11). In (12), the total load $P$ denotes the sum of the load over the solid and the fluid layers, Fig. 1.

\section{Onset of bifurcation}

The objectives of this section are to derive the conditions for the equilibrium of the fundamental solution and to detect the onset of bifurcation. The fundamental solution is the collection of equilibria for which the plate is flat as in the reference configuration, Fig. 1a.

The energy of the structure defined in (12) is interpreted as a function of only two independent field variables $v$ and $\omega$ since the constant $W$ is also defined in terms of these two variables in (11). Equilibrium at a given applied load requires the energy to be a minimum in terms of the displacement. This equilibrium statement requires that the Fréchet derivative of the energy vanishes

$$
\mathscr{E}(\mathbf{v})_{\mathbf{v}}[\widetilde{\mathbf{v}}]=0 \quad \forall \widetilde{\mathbf{v}} \in \mathscr{V} ; \quad v(0)=0, \omega_{, 1}(0)=\omega_{, 1}(L)=0,
$$

in which the vector $\mathbf{v}$ denotes the couple $(v, \omega)$ and $\mathscr{V}$ the set of admissible displacements denoted by $\widetilde{\mathbf{v}}$. Eq. (13) also includes the essential boundary conditions necessary to define the admissible displacements. The general equilibrium condition in (13) takes the particular form for the present problem

$$
\begin{aligned}
\mathscr{E}_{\mathbf{v}}[\widetilde{\mathbf{v}}]= & \int_{0}^{L}\left(N \epsilon_{\mathbf{v}}[\widetilde{\mathbf{v}}]+M \beta_{\mathbf{v}}[\widetilde{\mathbf{v}}]+H_{\mathrm{s}} \rho_{\mathrm{s}} \widetilde{\omega}+\rho_{\mathrm{f}} \omega\left(\widetilde{\omega}\left(1+v_{, 1}\right)+\frac{1}{2} \omega \widetilde{v}, 1\right)\right) \mathrm{d} X_{1}+P \widetilde{v}(L)+\rho_{\mathrm{s}} H_{\mathrm{s}} L W_{\mathbf{v}}[\widetilde{\mathbf{v}}] \\
& -\frac{1}{2} \rho_{\mathrm{f}}\left(2 W l W_{\mathbf{v}}[\widetilde{\mathbf{v}}]+\left(W^{2}+H_{\mathrm{f}}^{2}\right) \widetilde{v}(L)+2 W_{\mathbf{v}}[\widetilde{\mathbf{v}}] H_{\mathrm{f}} v(L)+2 H_{\mathrm{f}} W \widetilde{v}(L)\right)=0,
\end{aligned}
$$

in which a superposed tilde designates admissible quantities. The linear operators introduced in (14) are found from (2), (4) and (11) to be

$$
\begin{aligned}
& \epsilon_{\mathbf{v}}[\widetilde{\mathbf{v}}]=\lambda_{\mathbf{v}}[\widetilde{\mathbf{v}}]=\cos \psi \widetilde{v}_{, 1}+\sin \psi \widetilde{\omega}_{, 1}, \\
& \beta_{\mathbf{v}}[\widetilde{\mathbf{v}}] \lambda^{2}+2 \beta \lambda \lambda_{\mathbf{v}}[\widetilde{\mathbf{v}}]=\widetilde{\omega}_{, 1} v_{, 11}+\omega_{, 1} \widetilde{v}_{, 11}-\widetilde{\omega}_{, 11}\left(1+v_{, 1}\right)-\omega_{, 11} \widetilde{v}_{, 1}, \\
& W_{\mathbf{v}}[\widetilde{\mathbf{v}}] l=-\left(W+H_{\mathrm{f}}\right) \widetilde{v}(L)-\int_{0}^{L}\left(\widetilde{\omega}\left(1+v_{, 1}\right)+\omega \widetilde{v}_{, 1}\right) \mathrm{d} X_{1} .
\end{aligned}
$$

An updated Lagrangian formulation is now adopted to study the equilibria of the fundamental solution and the displacement and the strain measure are thus set to zero

$$
\mathbf{v}=0, \quad \mathbf{v}_{, 1}=0, \quad \psi=0, \quad \lambda=1, \quad \beta=0 \quad \forall X_{1} \in[0 ; L], W=0 .
$$

The linear operators found in (15) then simplify for this equilibrium solution to

$$
\epsilon_{\mathbf{v}}[\widetilde{\mathbf{v}}]=\lambda_{\mathbf{v}}[\widetilde{\mathbf{v}}]=\widetilde{v}_{, 1}, \quad \beta_{\mathbf{v}}[\widetilde{\mathbf{v}}]=-\widetilde{\omega}_{, 11}, \quad W_{\mathbf{v}}[\widetilde{\mathbf{v}}] L=-H_{\mathrm{f}} \widetilde{v}(L)-\int_{0}^{L} \widetilde{\omega} \mathrm{d} X_{1} .
$$


With this information, the minimization of the energy provides

$$
\int_{0}^{L} N^{\mathrm{p}}\left(\widetilde{v}_{, 1}-M^{\mathrm{p}} \widetilde{\omega}_{, 11}\right) \mathrm{d} X_{1}+\widetilde{v}(L)\left(P-\rho_{\mathrm{s}} H_{\mathrm{s}} H_{\mathrm{f}}-\frac{1}{2} \rho_{\mathrm{f}} H_{\mathrm{f}}^{2}\right)=0
$$

for a force control on the boundary. Integrating (18) by parts and accounting for the essential boundary conditions leads to an equation which, to be satisfied for any admissible $\widetilde{v}(L)$, necessitates in turns

$$
P=-N^{\mathrm{p}}+H_{\mathrm{f}}\left(H_{\mathrm{s}} \rho_{\mathrm{s}}+\frac{1}{2} \rho_{\mathrm{f}} H_{\mathrm{f}}\right) .
$$

One will recognize here the total force, positive in compression, necessary to balance the initial stress distribution in the plate and the weight of both the plate and the fluid.

The rest of this section is devoted to the onset of bifurcation from this collection of equilibria, one of them being the critical state at bifurcation which also defines the reference configuration. The absence of bifurcation is defined by the possibility to construct uniquely neighboring equilibrium states by displacing the boundary by $-\Delta$. This scalar, positive in compression $(l<L)$, is the "loading" parameter and the energy is now interpreted as a function of both $\mathbf{v}$ and $\Delta$. The collection of equilibria of the fundamental solution is given by

$$
v_{0}\left(X_{1}, \Delta\right)=-\Delta \frac{X_{1}}{L}, \quad \omega_{0}\left(X_{1}, \Delta\right)=0 \quad \text { and } \quad W_{0}(\Delta)=\frac{H_{\mathrm{f}} \Delta}{L-\Delta} .
$$

The searched equilibrium is constructed by a first-order development in terms of $\Delta$ of the equilibrium equations in (13)

$$
\mathscr{E}(\mathbf{v}, \Delta)_{\mathbf{v v}}[\widetilde{\mathbf{v}}, \widehat{\mathbf{v}}]+\mathscr{E}(\mathbf{v}, \Delta)_{\mathbf{v} \Delta}[\widetilde{\mathbf{v}}] \widehat{\Delta}=0 \quad \forall \widetilde{\mathbf{v}} \in \mathscr{V}
$$

for the loading increment $\widehat{\Delta}$ and the unknown displacement increment $\widehat{\mathbf{v}}$. This construction will fail as soon as the stability operator $\mathscr{E}_{\mathrm{vv}}$, defined as the second Fréchet derivative of the energy, has a zero eigenvalue with, for example, a single eigenvector $\mathbf{v}_{1}$ element of the null space $\mathscr{V}_{0}$. The critical initial normal force $N_{\mathrm{c}}^{\mathrm{p}}$ at which the first zero eigenvalue is detected defines the onset of bifurcation along the fundamental solution

$$
\exists N_{\mathrm{c}}^{\mathrm{p}} \text { such that } \mathscr{E}(\mathbf{v}=0, \Delta=0)_{\mathbf{v v}}\left[\widetilde{\mathbf{v}}, \mathbf{v}_{1}\right]=0 \quad \text { with }\left\|\mathbf{v}_{1}\right\|=1, \forall \widetilde{\mathbf{v}} \in \mathscr{V},
$$

where it is stressed that the stability operator is expressed on the selected reference configuration. Note that the letter c in subscript in (22), which could also be found in superscript in the rest of this paper, indicates that the operator is evaluated at the reference configuration assumed to be critical for bifurcation. It remains now to obtain the stability operator and to find the critical initial conditions for bifurcation ${ }^{1}$. For that purpose linearize the equilibrium conditions found in (14) knowing that the loading parameter $\Delta=-v(L)$ is held constant

$$
\begin{gathered}
\mathscr{E}_{\mathbf{v v}}[\widetilde{\mathbf{v}}, \widehat{\mathbf{v}}]=\int_{0}^{L}\left[N \epsilon_{\mathbf{v v}}[\widetilde{\mathbf{v}}, \widehat{\mathbf{v}}]+M \beta_{\mathbf{v v}}[\widetilde{\mathbf{v}}, \widehat{\mathbf{v}}]+E\left(\epsilon_{\mathbf{v}}[\widetilde{\mathbf{v}}] H_{\mathrm{s}} \epsilon_{\mathbf{v}}[\widehat{\mathbf{v}}]+\beta_{\mathbf{v}}[\widetilde{\mathbf{v}}] I \beta_{\mathbf{v}}[\widehat{\mathbf{v}}]\right)+\rho_{\mathrm{f}}\left(\widetilde{\omega}\left(\widehat{\omega}\left(1+v_{, 1}\right)+\omega \widehat{v}_{, 1}\right)\right.\right. \\
\left.\left.+\omega \widehat{\omega} \widetilde{v}_{, 1}\right)\right] \mathrm{d} X_{1}-\rho_{\mathrm{f}} l\left(W_{\mathbf{v}}[\widehat{\mathbf{v}}] W_{\mathbf{v}}[\widetilde{\mathbf{v}}]+W W_{\mathbf{v v}}[\widetilde{\mathbf{v}}, \widehat{\mathbf{v}}]\right)+\left(\rho_{\mathrm{f}} \Delta H_{\mathrm{f}}+H_{\mathrm{s}} \rho_{\mathrm{s}} L\right) W_{\mathbf{v v}}[\widetilde{\mathbf{v}}, \widehat{\mathbf{v}}]=0,
\end{gathered}
$$

in which the operator $\epsilon_{\mathbf{v v}}, \beta_{\mathbf{v v}}$ and $W_{\mathbf{v v}}$ are found from (17) to be

\footnotetext{
${ }^{1}$ To differentiate between limit load and bifurcation, one should also check that $\mathscr{E}(\mathbf{v}=0, \Delta=0)_{\mathbf{v} \Delta}\left[\mathbf{v}_{1}\right]$ is zero (Triantafyllidis and Peek, 1992), a condition also respected here.
} 


$$
\begin{aligned}
\epsilon_{\mathbf{v v}}[\widetilde{\mathbf{v}}, \widehat{\mathbf{v}}] \lambda=- & \lambda_{\mathbf{v}}[\widehat{\mathbf{v}}] \lambda_{\mathbf{v}}[\widetilde{\mathbf{v}}]+\widetilde{v}_{, 1} \widehat{v}_{, 1}+\widetilde{\omega}_{, 1} \widehat{\omega}_{, 1}, \\
\beta_{\mathbf{v v}}[\widetilde{\mathbf{v}}, \widehat{\mathbf{v}}] \lambda^{2}=- & -2 \beta_{\mathbf{v}}[\widetilde{\mathbf{v}}] \lambda \lambda_{\mathbf{v}}[\widehat{\mathbf{v}}]-2 \beta_{\mathbf{v}}[\widehat{\mathbf{v}}] \lambda \lambda_{\mathbf{v}}[\widetilde{\mathbf{v}}]-2 \beta\left(\lambda_{\mathbf{v}}[\widetilde{\mathbf{v}}] \lambda_{\mathbf{v}}[\widehat{\mathbf{v}}]+\lambda \lambda_{\mathbf{v v}}[\widetilde{\mathbf{v}}, \widehat{\mathbf{v}}]\right) \\
& +\widetilde{\omega}_{, 1} \widehat{v}_{, 11}+\widehat{\omega}_{, 1} \widetilde{v}_{, 11}-\widetilde{\omega}_{, 11} \widehat{v}_{, 1}-\widehat{\omega}_{, 11} \widetilde{v}_{, 1}, \\
W_{\mathbf{v v}}[\widetilde{\mathbf{v}}, \widehat{\mathbf{v}}] l=- & \int_{0}^{L} \widetilde{\omega} \widehat{v}_{, 1}+\widehat{\omega} \widetilde{v}_{, 1} \mathrm{~d} X_{1} .
\end{aligned}
$$

The next step consists of specializing the operators in (24) and Eq. (23) to the reference state described by (16) and (17), to incorporate the former in the latter to obtain

$$
\begin{gathered}
\mathscr{E}_{\mathbf{v v}}^{\mathfrak{c}}[\widetilde{\mathbf{v}}, \widehat{\mathbf{v}}]=\int_{0}^{L}\left[N^{\mathrm{p}} \widehat{\omega}_{, 1} \widetilde{\omega}_{, 1}+M^{\mathrm{p}}\left(\widehat{\omega}_{, 1} \widetilde{v}_{, 11}+\widetilde{\omega}_{, 1} \widehat{v}_{, 11}+\widehat{\omega}_{, 11} \widetilde{v}_{, 1}+\widetilde{\omega}_{, 11} \widehat{v}_{, 1}\right)+E\left(\widetilde{v}_{, 1} H_{\mathrm{s}} \widehat{v}_{, 1}+\widehat{\omega}_{, 11} I \widetilde{\omega}_{, 11}\right)\right. \\
\left.+\rho_{\mathrm{f}} \widetilde{\omega} \widehat{\omega}\right] \mathrm{d} X_{1}-\rho_{\mathrm{f}} W_{\mathbf{v}}[\widehat{\mathbf{v}}] L W_{\mathbf{v}}[\widetilde{\mathbf{v}}]+H_{\mathrm{s}} \rho_{\mathrm{s}} L W_{\mathbf{v v}}[\widetilde{\mathbf{v}}, \widehat{\mathbf{v}}]=0 .
\end{gathered}
$$

One needs now to integrate by parts the first and second derivatives of the admissible displacement to obtain an integral equation with two terms multiplied by $\widetilde{v}$ and $\widetilde{\omega}$. Accounting for the essential boundary conditions $(\widetilde{v}(0)=\widetilde{v}(L)=0)$, the Euler-Lagrange equations with boundary conditions are then found to be

$$
\begin{aligned}
& E I \widehat{\omega}_{, 111}-N^{\mathrm{p}} \widehat{\omega}_{, 11}+\rho_{\mathrm{f}}(\widehat{\omega}+\widehat{W})-\rho_{\mathrm{s}} H_{\mathrm{s}} \widehat{v}_{, 1}=0, \\
& E \widehat{v}_{, 11}-\rho_{\mathrm{s}} \widehat{\omega}_{, 1}=0 \quad \forall X_{1} \in[0 ; L] \quad \text { with } \widehat{v}=\widehat{\omega}_{, 1}=\widehat{\omega}_{, 111}=0 \text { at } X_{1}=0, L .
\end{aligned}
$$

Resolution of this system of equations is standard and provides the critical normal force

$$
N_{\mathrm{c}}^{\mathrm{p}}=-E I p^{2}-\frac{1}{p^{2}}\left(\rho_{\mathrm{f}}-\rho_{\mathrm{s}}^{2} \frac{H_{\mathrm{s}}}{E}\right) \quad \text { with } p=\frac{n \pi}{L} \text { and } n \in \mathbb{N}^{*}
$$

and the eigenvector defined in (22)

$$
\omega_{1}\left(X_{1}\right)=\cos p X_{1}, \quad W_{1}=0, \quad v_{1}\left(X_{1}\right)=\frac{\rho_{\mathrm{s}}}{E} \frac{1}{p} \sin p X_{1} .
$$

Note that the decomposition of the eigenmode in terms of $\omega_{1}$ and $W_{1}$ is not unique but the sum of the two is. Note also that the amplitude of the eigenmode in (28) has not been specified since the normalization proposed in (22) has not been applied for sake of simplicity. This absence has no consequence on the asymptotic calculations presented next.

\section{Asymptotic construction of the bifurcated solution}

The conclusion of the previous section is that there is a critical initial stress condition from which the fundamental solution cannot be constructed uniquely in terms of the control parameter. One needs now to establish the existence of a second equilibrium solution and the accepted approach for conservative systems is the Lyapunov-Schmidt-Koiter method (e.g., Triantafyllidis and Peek, 1992). The equilibrium solution is constructed from the addition of two parts, the first being the fundamental solution $\mathbf{v}_{0}$ and $W_{0}$ given in (20). The second part of the solution is also partitioned into two contributions. The first is proportional to the stability mode $\xi \mathbf{v}_{1}$ in the null space $\mathscr{V}_{0}$, the scalar $\xi$ being the bifurcation amplitude coefficient. The second contribution is orthogonal to the null space and admits an asymptotic development in terms of the scalar $\xi$ with $\mathbf{v}_{2}\left(X_{1}\right)$ being the first non-trivial term of that expansion. This function and higher-order displacement functions satisfy the orthogonality condition

$$
\int_{0}^{L} \mathbf{v}_{1} \cdot \mathbf{v}_{\alpha} \mathrm{d} X_{1}=0 \quad \forall \alpha \geqslant 2
$$


Combining these two contributions and the first part based on the fundamental solution provides the structure of the post-bifurcation solution in the form of an asymptotic development

$$
\mathbf{v}\left(X_{1}, \Delta\right)=\mathbf{v}_{0}\left(X_{1}, \Delta\right)+\xi \mathbf{v}_{1}\left(X_{1}\right)+\xi^{2} \frac{1}{2} \mathbf{v}_{2}\left(X_{1}\right)+\cdots,
$$

in which the small parameter $\xi$, the bifurcation amplitude coefficient, is defined by

$$
\xi=\frac{\int_{0}^{L} \mathbf{v}_{1} \cdot\left(\mathbf{v}-\mathbf{v}_{0}\right) \mathrm{d} X_{1}}{\int_{0}^{L} \mathbf{v}_{1} \cdot \mathbf{v}_{1} \mathrm{~d} X_{1}},
$$

thanks to the orthogonality condition (29), and has for dimension length. A similar development to (30) is proposed for the loading parameter $\Delta$

$$
\Delta=\xi \Delta_{1}+\xi^{2} \frac{1}{2} \Delta_{2}+\cdots,
$$

where the condition that $\Delta$ is zero at criticality has already been accounted for. The problem thus consists of determining the two scalars $\Delta_{1}, \Delta_{2}$ and the displacement function $\mathbf{v}_{2}\left(X_{1}\right)$ introduced in (30). By enforcing equilibrium in the null space and in its supplement in the set of admissible displacements one obtains three equations

$$
\begin{aligned}
& \Delta_{1}=-\frac{1}{2} \mathscr{E}_{\mathbf{v v v}}^{\mathrm{c}}\left[\mathbf{v}_{1}, \mathbf{v}_{1}, \mathbf{v}_{1}\right] /\left(\frac{\mathrm{d}}{\mathrm{d} \Delta} \mathscr{E}_{\mathbf{v v}}\left[\mathbf{v}_{1}, \mathbf{v}_{1}\right]\right)^{\mathrm{c}} \\
& \mathscr{E}_{\mathbf{v v}}^{\mathrm{c}}\left[\widetilde{\mathbf{v}}, \mathbf{v}_{2}\right]+\mathscr{E}_{\mathbf{v v v}}^{\mathrm{c}}\left[\widetilde{\mathbf{v}}, \mathbf{v}_{1}, \mathbf{v}_{1}\right]=0 \quad \forall \widetilde{\mathbf{v}} \in \mathscr{V}, \\
& \Delta_{2}=-\frac{1}{3}\left(\mathscr{E}_{\mathbf{v v v}}^{\mathscr{c}}\left[\mathbf{v}_{1}, \mathbf{v}_{1}, \mathbf{v}_{1}, \mathbf{v}_{1}\right]-3 \mathscr{E}_{\mathbf{v v}} \mathrm{c}\left[\mathbf{v}_{2}, \mathbf{v}_{2}\right]\right) /\left(\frac{\mathrm{d}}{\mathrm{d} \Delta} \mathscr{E}_{\mathbf{v v}}\left[\mathbf{v}_{1}, \mathbf{v}_{1}\right]\right)^{\mathrm{c}}
\end{aligned}
$$

necessary to determine these unknowns.

It can be seen, without any calculation, that the first of the three unknowns, $\Delta_{1}$ is identical to zero. This conclusion is reached by replacing the eigenmode $\mathbf{v}_{1}$ by its opposite $-\mathbf{v}_{1}$ in the development (30). The structure being symmetric with respect to the vertical axis passing through $X_{1}=L / 2$, the selection of $\mathbf{v}_{1}$ or $-\mathbf{v}_{1}$ cannot influence the evolution of the displacement at the boundary and thus the scalar $\Delta_{1}$ in (32) has to be zero.

The rest of this section is divided into two parts to present first the solution $\mathbf{v}_{2}$ to the second equation in (33) and second, the evaluation of the three terms contributing to the value of $\Delta_{2}$ in the third equation in (33).

\subsection{The second-order displacement function $\mathbf{v}_{2}$}

The second equation in (33) is a statement of equilibrium in the supplementary part of the null space in $\mathscr{V}$. The first term appearing in the left-hand side is the second Fréchet derivative of the energy at the critical load and is identical to (25) with $\widehat{\mathbf{v}}$ replaced by $\mathbf{v}_{2}$. The treatment of this term thus does not require further discussion and will lead to the two ordinary differential equations as in (26). These equations are complemented by the contribution of the second term in (33) which is the third Fréchet derivative of the energy. This derivative is constructed from (23) and reads

$$
\begin{aligned}
\mathscr{E}_{\mathbf{v v v}}[\widetilde{\mathbf{v}}, \widehat{\mathbf{v}}, \overline{\mathbf{v}}]= & \int_{0}^{L}\left[N \epsilon_{\mathbf{v v v}}[\widetilde{\mathbf{v}}, \widehat{\mathbf{v}}, \overline{\mathbf{v}}]+E H_{\mathrm{s}}\left(\epsilon_{\mathbf{v v}}[\widetilde{\mathbf{v}}, \overline{\mathbf{v}}] \epsilon_{\mathbf{v}}[\widehat{\mathbf{v}}]+\epsilon_{\mathbf{v v}}[\widehat{\mathbf{v}}, \overline{\mathbf{v}}] \epsilon_{\mathbf{v}}[\widetilde{\mathbf{v}}]+\epsilon_{\mathrm{vv}}[\widetilde{\mathbf{v}}, \widehat{\mathbf{v}}] \epsilon_{\mathbf{v}}[\overline{\mathbf{v}}]\right)+M \beta_{\mathrm{vvv}}[\widetilde{\mathbf{v}}, \widehat{\mathbf{v}}, \overline{\mathbf{v}}]\right. \\
& \left.+E I\left(\beta_{\mathbf{v v}}[\widetilde{\mathbf{v}}, \overline{\mathbf{v}}] \beta_{\mathbf{v}}[\widehat{\mathbf{v}}]+\beta_{\mathbf{v v}}[\widehat{\mathbf{v}}, \overline{\mathbf{v}}] \beta_{\mathbf{v}}[\widetilde{\mathbf{v}}]+\beta_{\mathbf{v v}}[\widetilde{\mathbf{v}}, \widehat{\mathbf{v}}] \beta_{\mathbf{v}}[\overline{\mathbf{v}}]\right)+\rho_{\mathrm{f}}\left(\widetilde{\omega} \widehat{\omega} \bar{v}_{, 1}+\widetilde{\omega} \bar{\omega} \widehat{v}_{, 1}+\bar{\omega} \widehat{\omega} \widetilde{v}, 1\right)\right] \mathrm{d} X_{1} \\
& -\rho_{\mathrm{f}}\left(W_{\mathbf{v v}}[\widehat{\mathbf{v}}, \overline{\mathbf{v}}] l W_{\mathbf{v}}[\widetilde{\mathbf{v}}]+W_{\mathbf{v v}}[\widetilde{\mathbf{v}}, \overline{\mathbf{v}}] l W_{\mathbf{v}}[\widehat{\mathbf{v}}]+W_{\mathbf{v v}}[\widehat{\mathbf{v}}, \widetilde{\mathbf{v}}] l W_{\mathbf{v}}[\overline{\mathbf{v}}]\right),
\end{aligned}
$$


in which the two linear operators $\epsilon_{\mathbf{v v v}}[\widetilde{\mathbf{v}}, \widehat{\mathbf{v}}, \overline{\mathbf{v}}]$ and $\beta_{\mathbf{v v v}}[\widetilde{\mathbf{v}}, \widehat{\mathbf{v}}, \overline{\mathbf{v}}]$ are found from (24) to be

$$
\begin{aligned}
\epsilon_{\mathbf{v v v}}[\widetilde{\mathbf{v}}, \widehat{\mathbf{v}}, \overline{\mathbf{v}}] \lambda= & -\lambda_{\mathrm{v}}[\widehat{\mathbf{v}}] \lambda_{\mathrm{vv}}[\widetilde{\mathbf{v}}, \overline{\mathbf{v}}]-\lambda_{\mathrm{v}}[\overline{\mathbf{v}}] \lambda_{\mathrm{vv}}[\widetilde{\mathbf{v}}, \widehat{\mathbf{v}}]-\lambda_{\mathrm{v}}[\widetilde{\mathbf{v}}] \lambda_{\mathrm{vv}}[\widehat{\mathbf{v}}, \overline{\mathbf{v}}] \\
\beta_{\mathrm{vvv}}[\widetilde{\mathbf{v}}, \widehat{\mathbf{v}}, \overline{\mathbf{v}}] \lambda^{2}= & -2 \beta_{\mathrm{vv}}[\widetilde{\mathbf{v}}, \overline{\mathbf{v}}] \lambda \lambda_{\mathrm{v}}[\widehat{\mathbf{v}}]-2 \beta_{\mathrm{vv}}[\widehat{\mathbf{v}}, \overline{\mathbf{v}}] \lambda \lambda_{\mathrm{v}}[\widetilde{\mathbf{v}}]-2 \beta_{\mathrm{vv}}[\widetilde{\mathbf{v}}, \widehat{\mathbf{v}}] \lambda \lambda_{\mathrm{v}}[\overline{\mathbf{v}}] \\
& -2 \beta\left(\lambda_{\mathrm{v}}[\widehat{\mathbf{v}}] \lambda_{\mathrm{vv}}[\widetilde{\mathbf{v}}, \overline{\mathbf{v}}]+\lambda_{\mathrm{v}}[\widetilde{\mathbf{v}}] \lambda_{\mathrm{vv}}[\widehat{\mathbf{v}}, \overline{\mathbf{v}}]+\lambda_{\mathrm{v}}[\overline{\mathbf{v}}] \lambda_{\mathrm{vv}}[\widetilde{\mathbf{v}}, \widehat{\mathbf{v}}]\right) \\
& -2 \beta_{\mathrm{v}}[\widehat{\mathbf{v}}]\left(\lambda_{\mathrm{v}}[\widetilde{\mathbf{v}}] \lambda_{\mathrm{v}}[\overline{\mathbf{v}}]+\lambda \lambda_{\mathrm{vv}}[\widetilde{\mathbf{v}}, \overline{\mathbf{v}}]\right)-2 \beta_{\mathrm{v}}[\widetilde{\mathbf{v}}]\left(\lambda_{\mathrm{v}}[\widehat{\mathbf{v}}] \lambda_{\mathrm{v}}[\overline{\mathbf{v}}]+\lambda \lambda_{\mathrm{vv}}[\widehat{\mathbf{v}}, \overline{\mathbf{v}}]\right) \\
& -2 \beta_{\mathrm{v}}[\overline{\mathbf{v}}]\left(\lambda_{\mathrm{v}}[\widetilde{\mathbf{v}}] \lambda_{\mathrm{v}}[\widehat{\mathbf{v}}]+\lambda \lambda_{\mathrm{vv}}[\widetilde{\mathbf{v}}, \widehat{\mathbf{v}}]\right) .
\end{aligned}
$$

Note that the operator $W_{\mathrm{vv}}$ is identical to zero, explaining its absence from (34). The next step consists of specializing (34) and (35) to the reference state defined by (16) and (17) with the displacement $\widehat{\mathbf{v}}$ and $\overline{\mathbf{v}}$ set to the eigenmode $\mathbf{v}_{1}$ to obtain

$$
\begin{aligned}
\mathscr{E}_{\mathbf{v v v}}^{\mathrm{c}}\left[\widetilde{\mathbf{v}}, \mathbf{v}_{1}, \mathbf{v}_{1}\right]= & \int_{0}^{L}\left[\widetilde{\omega}_{, 11}\left(M^{\mathrm{p}} 2\left(S_{1}^{2} p^{2}-C_{1}^{2}\left(\frac{\rho_{\mathrm{s}}}{E}\right)^{2}\right)+I \rho_{\mathrm{s}} p^{2} 2\left(2 C_{1}^{2}-S_{1}^{2}\right)\right)\right. \\
& +\widetilde{\omega}_{, 1}\left(N^{\mathrm{p}} \frac{\rho_{\mathrm{s}}}{E}+M^{\mathrm{p}} 2\left(p^{2}+\left(\frac{\rho_{\mathrm{s}}}{E}\right)^{2}\right)-H_{\mathrm{s}} E \frac{\rho_{\mathrm{s}}}{E}-E I p^{2}\right) C_{1} S_{1} 2 p+\widetilde{\omega}\left(C_{1}^{2}-S_{1}^{2}\right) \frac{\rho_{\mathrm{s}} \rho_{\mathrm{f}}}{E} \\
& -\widetilde{v}_{, 11}\left(2 p^{2} I E-\frac{\rho_{\mathrm{s}}}{E} 4 M^{\mathrm{p}}\right) C_{1} S_{1} p+\widetilde{v}_{, 1}\left(-N^{\mathrm{p}} S_{1}^{2} p^{2}+M^{\mathrm{p}} 4 \frac{\rho_{\mathrm{s}}}{E} p^{2}\left(C_{1}^{2}-S_{1}^{2}\right)+H_{\mathrm{s}} E S_{1}^{2} p^{2}\right. \\
& \left.\left.-I E 2 C_{1}^{2} p^{4}+\rho_{\mathrm{f}} C_{1}^{2}\right)\right] \mathrm{d} X_{1},
\end{aligned}
$$

in which $C_{1}$ and $S_{1}$ denotes $\cos \left(p X_{1}\right)$ and $\sin \left(p X_{1}\right)$, respectively. Integrating by parts to eliminate the derivative of the admissible displacements and accounting for the essential boundary conditions, one obtains the searched contribution to the two ordinary differential equations which govern the second-order displacement function $\mathbf{v}_{2}$. These two equations are

$$
\begin{aligned}
& E I \omega_{2,1111}-N^{\mathrm{p}} \omega_{2,11}+\rho_{\mathrm{f}}\left(\omega_{2}+W_{2}\right)-\rho_{\mathrm{s}} H_{\mathrm{s}} v_{2,1}+\frac{\rho_{\mathrm{s}}}{E}\left(-8 E I p^{4}+3 \rho_{\mathrm{f}}+2 \frac{H_{\mathrm{s}}}{E}\left(-\rho_{\mathrm{s}}^{2}+E^{2} p^{2}\right)\right) C_{2}=0, \\
& v_{2,11}-\frac{\rho_{\mathrm{s}}}{E} \omega_{2,1}-\frac{1}{E H_{\mathrm{s}}}\left(E I p^{5}+p \frac{H_{\mathrm{s}}}{E}\left(\rho_{\mathrm{s}}^{2}-E^{2} p^{2}\right)\right) S_{2}=0 \quad \forall X_{1} \in[0 ; L] \quad \text { with } \\
& v_{2}=\omega_{2,1}=\omega_{2,111}=0 \text { at } X_{1}=0, L,
\end{aligned}
$$

in which $C_{2}$ and $S_{2}$ denotes $\cos \left(2 p X_{1}\right)$ and $\sin \left(2 p X_{1}\right)$, respectively. The solutions to (37) are

$$
\omega_{2}=\stackrel{2}{\omega} C_{2}, \quad W_{2}=0, \quad V_{2}=\stackrel{2}{v} S_{2},
$$

in which the amplitudes $\stackrel{2}{\omega}$ and ${ }^{2}$ are found from the orthogonality conditions (29)

$$
\stackrel{2}{\omega}=\frac{\rho_{\mathrm{s}}}{2 E} \frac{5 p^{4} E I-2 \rho_{\mathrm{f}}+E H_{\mathrm{s}}\left(\left(\frac{\rho_{\mathrm{s}}}{E}\right)^{2}-p^{2}\right)}{4 p^{4} E I-\rho_{\mathrm{f}}+\frac{H_{\mathrm{s}}}{E} \rho_{\mathrm{s}}^{2}}, \quad \stackrel{2}{v}=-\frac{p^{3} I}{4 H_{\mathrm{s}}}+\frac{1}{4 p}\left(p^{2}-\left(\frac{\rho_{\mathrm{s}}}{E}\right)^{2}\right)+\frac{\rho_{\mathrm{s}}}{2 E p} \stackrel{2}{\omega} .
$$

\subsection{The second-order control parameter $\Delta_{2}$}

The last task of this section concerns the computation of the scalar $\Delta_{2}$ defined in (33) in terms of three derivatives of the energy which are now documented. The first of the three is the fourth Fréchet derivative of the energy which is found by taking the derivative of (34) and applying the resulting multilinear form to the eigenmode $\mathbf{v}_{1}$ 


$$
\begin{aligned}
\mathscr{E}_{\mathbf{v v v v}}\left[\mathbf{v}_{1}, \mathbf{v}_{1}, \mathbf{v}_{1}, \mathbf{v}_{1}\right]= & \int_{0}^{L}\left[N \epsilon_{\mathbf{v v v v}}\left[\mathbf{v}_{1}, \mathbf{v}_{1}, \mathbf{v}_{1}, \mathbf{v}_{1}\right]+E H_{\mathrm{s}}\left(4 \epsilon_{\mathbf{v v v}}\left[\mathbf{v}_{1}, \mathbf{v}_{1}, \mathbf{v}_{1}\right] \epsilon_{\mathbf{v}}\left[\mathbf{v}_{1}\right]+3\left(\epsilon_{\mathbf{v v}}\left[\mathbf{v}_{1}, \mathbf{v}_{1}\right]\right)^{2}\right)\right] \\
& +\left[M \beta_{\mathbf{v v v}}\left[\mathbf{v}_{1}, \mathbf{v}_{1}, \mathbf{v}_{1}, \mathbf{v}_{1}\right]+E I\left(4 \beta_{\mathbf{v v v}}\left[\mathbf{v}_{1}, \mathbf{v}_{1}, \mathbf{v}_{1}\right] \beta_{\mathbf{v}}\left[\mathbf{v}_{1}\right]+3 \beta_{\mathbf{v v}}\left[\mathbf{v}_{1}, \mathbf{v}_{1}\right]^{2}\right)\right] \mathrm{d} X_{1} \\
& -\rho_{\mathrm{f}} 3 l\left(W_{\mathbf{v v}}\left[\mathbf{v}_{1}, \mathbf{v}_{1}\right]\right)^{2},
\end{aligned}
$$

in which the two quadrilinear operators introduced above are defined by

$$
\begin{aligned}
& \epsilon_{\mathrm{vvvv}}\left[\mathbf{v}_{1}, \mathbf{v}_{1}, \mathbf{v}_{1}, \mathbf{v}_{1}\right] \lambda=-3 \lambda_{\mathrm{vvv}}\left[\mathbf{v}_{1}, \mathbf{v}_{1}, \mathbf{v}_{1}\right] \lambda \lambda_{\mathrm{v}}\left[\mathbf{v}_{1}\right]-4 \lambda_{\mathrm{vv}}\left[\mathbf{v}_{1}, \mathbf{v}_{1}\right] \lambda_{\mathrm{vv}}\left[\mathbf{v}_{1}, \mathbf{v}_{1}\right], \\
& \beta_{\mathrm{vvvv}}\left[\mathbf{v}_{1}, \mathbf{v}_{1}, \mathbf{v}_{1}, \mathbf{v}_{1}\right] \lambda^{2}=-8 \beta_{\mathrm{vvv}}\left[\mathbf{v}_{1}, \mathbf{v}_{1}, \mathbf{v}_{1}\right] \lambda \lambda_{\mathrm{v}}\left[\mathbf{v}_{1}\right]-12 \beta_{\mathrm{vv}}\left[\mathbf{v}_{1}, \mathbf{v}_{1}\right]\left(\lambda \lambda_{\mathrm{v}}\right)_{\mathbf{v}}\left[\mathbf{v}_{1}, \mathbf{v}_{1}\right] .
\end{aligned}
$$

Note that the second expression above accounts for the fact that the operator $\left(\lambda \lambda_{\mathbf{v}}\right)_{\mathbf{v v}}\left[\mathbf{v}_{1}, \mathbf{v}_{1}, \mathbf{v}_{1}\right]$ and $\left(\lambda \lambda_{\mathbf{v}}\right)_{\mathbf{v v v}}\left[\mathbf{v}_{1}, \mathbf{v}_{1}, \mathbf{v}_{1}\right]$ are identical to zero. Eq. (40) is now specialized to the critical reference configuration accounting for the expression of the eigenmode $\mathbf{v}_{1}$ found in (28) and it becomes after integration

$$
\mathscr{E}_{\mathbf{v v v v}}^{\mathfrak{c}}\left[\mathbf{v}_{1}, \mathbf{v}_{1}, \mathbf{v}_{1}, \mathbf{v}_{1}\right]=\left(-N^{\mathrm{p}}+H_{\mathrm{s}} E\right) L p^{2} \frac{9}{8}\left(p^{2}-\frac{4}{3}\left(\frac{\rho_{\mathrm{s}}}{E}\right)^{2}\right)-\rho_{\mathrm{f}} 3 L\left(\frac{\rho_{\mathrm{s}}}{E}\right)^{2}-I E p^{4} L 3\left(p^{2}-3\left(\frac{\rho_{\mathrm{s}}}{E}\right)^{2}\right) .
$$

The second term of interest here to compute $\Delta_{2}$ in $(33 \mathrm{c})$ is the second Fréchet derivative $\mathscr{E}_{\mathrm{vv}}\left[\mathbf{v}_{2}, \mathbf{v}_{2}\right]$ in terms of the second-order displacement function. The starting point for its computation is (23) which is then particularized to the reference state with the explicit expression for the second-order displacement function found in (38). The end result after integration is

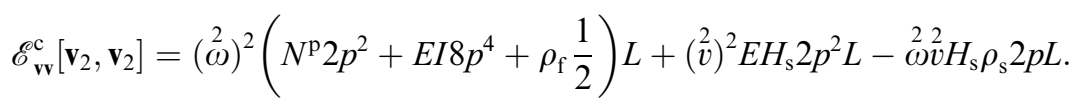

The last term required to compute $\Delta_{2}$ is the denominator of (33)c and consists of $\left(\mathrm{d} / \mathrm{d} \Delta \mathscr{E}_{\mathbf{v v}}\left[\mathbf{v}_{1}, \mathbf{v}_{1}\right]\right)^{\mathrm{c}}$ which is the derivative with respect to the loading parameter of the second Fréchet derivative of the energy along the fundamental solution. The value of the derivative is taken at the critical point and for the eigenmode $\mathbf{v}_{1}$. The second Fréchet derivative of the energy along this fundamental solution beyond the reference state is found from (23)

$$
\begin{aligned}
\mathscr{E}_{\mathbf{v v}}\left[\mathbf{v}_{1}, \mathbf{v}_{1}\right]=\int_{0}^{L} & {\left[\left(N^{\mathrm{p}}-H_{\mathrm{s}} E \frac{\Delta}{L}\right) \omega_{1,1}^{2}\left(1-\frac{\Delta}{L}\right)^{-1}+\rho_{\mathrm{f}} \omega_{1}^{2}\left(1-\frac{\Delta}{L}\right)+2 M^{\mathrm{p}}\left(\omega_{1,11} v_{1,1}+\omega_{1,1} v_{1,11}\right)\right.} \\
& \left.\times\left(1-\frac{\Delta}{L}\right)^{-2}+E H_{\mathrm{s}} v_{1,1}^{2}+E I \omega_{1,1}^{2}\left(1-\frac{\Delta}{L}\right)^{-2}-\frac{2 L}{L-\Delta} \rho_{\mathrm{s}} H_{\mathrm{s}} \omega_{1} v_{1,1}\right] \mathrm{d} X_{1},
\end{aligned}
$$

with the linear and bilinear operators defined in (15) and (24) estimated with (20). Taking the derivative of (44) provides

$$
\left(\frac{\mathrm{d}}{\mathrm{d} \Delta} \mathscr{E}_{\mathrm{vv}}\left[\mathbf{v}_{1}, \mathbf{v}_{1}\right]\right)^{\mathrm{c}}=\frac{1}{2} E I p^{4}-\rho_{\mathrm{f}}-\frac{1}{2} E H_{\mathrm{s}} p^{2}-\frac{1}{2} \rho_{\mathrm{s}} \frac{\rho_{\mathrm{s}} H_{\mathrm{s}}}{E}
$$

once the first-order eigenmode has been explicitly introduced, the integration over the domain has been done and the expression has been evaluated at the critical reference configuration.

Having computed the three contributions to the scalar $\Delta_{2}$, we are now ready to analyze the post-bifurcation solutions for a particular example. Before doing so, the expression for the normal forces $P_{\mathrm{s}}$ and $P_{\mathrm{f}}$, applied at the boundary of the plate and of the fluid respectively, as well as the total force $P$ are discussed. The force on the plate $P_{\mathrm{s}}$ is $-N_{\mathrm{c}}^{\mathrm{p}}-E H_{\mathrm{s}} \epsilon\left(X_{1}=L\right)$ and is now expressed in terms of the asymptotic development discussed above as follows. The definition of the strain $\epsilon$ in (4), of the fundamental solution (20), of 
the asymptotic development (30) and (32) are combined with the eigenmode and displacement function in (28), (38) and (39) to obtain

$$
P_{\mathrm{s}}=-N_{\mathrm{c}}^{\mathrm{p}}+E H_{\mathrm{s}}\left(\xi \frac{\rho_{\mathrm{s}}}{E}+\xi^{2}\left(\frac{\Delta_{2}}{2 L}-\stackrel{2}{v p}\right)\right)+\cdots
$$

The total force $P$ conjugate to the control parameter $\Delta$ could be computed from (14) knowing the asymptotic structure of the bifurcated solution. A more pragmatic approach is proposed starting with the calculation of the force applied to the fluid layer on the right boundary of the structure. The total weight of the structure being preserved by the transformation, one estimates the pressure at the bottom of the structure and, from the gradient in the fluid layer, determines the force for any current configuration

$$
P_{\mathrm{f}}=\left(H_{\mathrm{f}}+w(L)\right)\left(\left(\rho_{\mathrm{s}} H_{\mathrm{s}}+\rho_{\mathrm{f}} H_{\mathrm{f}}\right) \frac{L}{L-\Delta}-\frac{1}{2} \rho_{\mathrm{f}}\left(w(L)+H_{\mathrm{f}}\right)\right) .
$$

The asymptotic development is now incorporated in (47), following a similar procedure as for deriving (46), resulting in

$$
P_{\mathrm{f}}=\rho_{\mathrm{s}} H_{\mathrm{s}} H_{\mathrm{f}}+\frac{1}{2} \rho_{\mathrm{f}} H_{\mathrm{f}}^{2}-H_{\mathrm{s}} \rho_{\mathrm{s}} \xi+\frac{1}{2} \xi^{2}\left(-\rho_{\mathrm{f}} H_{\mathrm{f}}^{2} \frac{\Delta_{2}}{L}+\stackrel{2}{\omega} \rho_{\mathrm{s}} H_{\mathrm{s}}-\rho_{\mathrm{f}}\right)+\mathcal{O}\left(\xi^{3}\right) .
$$

The total force on the boundary is then simply the sum of $P_{\mathrm{s}}$ and $P_{\mathrm{f}}$ in (46) and (48) and reads

$$
P=P_{\mathrm{c}}+\frac{1}{2} \xi^{2}\left(\left(E H_{\mathrm{s}}-\rho_{\mathrm{f}} H_{\mathrm{f}}^{2}\right) \frac{\Delta_{2}}{L}+\stackrel{2}{\omega} \rho_{\mathrm{s}} H_{\mathrm{s}}-2 E H_{\mathrm{s}} v p-\rho_{\mathrm{f}}\right)+\mathcal{O}\left(\xi^{3}\right)
$$

in which $P_{\mathrm{c}}$ is the total force at bifurcation. The expressions for $P_{\mathrm{s}}, P_{\mathrm{f}}$ and $P$, presented in (46), (48) and (49), deserve some comments. The forces on the solid and the fluid layer have a leading term of order $\xi$ which are characteristic of asymmetric bifurcations. For example, the force on the solid plate would not increase but decrease in the post-buckling regime if the bifurcation amplitude coefficient was changed from $+\xi$ to $-\xi$. It is the opposite influence which is observed for the force applied of the fluid part of the structure. Moreover, the leading term in $\xi$ for the solid (46) and the fluid (48) are the same but of opposite sign. As a consequence the leading term for the total force in (49) is of second-order in $\xi$ since the sum of these two first-order contributions cancelled out. It thus remains to determine the sign of the leading term in (49) to determine if the total force will increase or decrease during the post-bifurcation which is symmetric in terms of $\xi$. This question is examined in the next section for a particular example which justify some simplifying assumptions.

\section{Application}

The discussion of the asymptotic construction of the equilibrium path is conducted for a specific application, a folded section in the Campos basin, offshore Brazil, presented by Cobbold and Szatmari (1991). The onset of folding has been studied by Triantafyllidis and Leroy (1997) assuming an elasto-plastic rheology and the data required for the present discussion is summarized in Table 1.

The normalized critical load over the elastic plate for the onset of folding is expressed by (27) and is plotted in Fig. 2 as a function of $p=n \pi / L$. Two additive contributions result in the expression for the critical load which are plotted as dotted and dashed curves in Fig. 2. The first contribution is due to the elastic stiffness of the plate and results in the classical quadratic expression for the Euler load (dotted curve). The longer the plate, the smaller is the scalar $p$ and the magnitude of the critical load to initiate buckling in the absence of the fluid. It is the action of gravity on the fluid which penalizes the long-wavelength mode 
Table 1

The geometry and elastic properties for the layered structure

\begin{tabular}{llll}
\hline Parameter & Definition & Value & Unit \\
\hline$H_{\mathrm{s}}$ & Solid plate thickness & 250 & $\mathrm{~m}$ \\
$H_{\mathrm{f}}$ & Fluid layer thickness & 600 & $\mathrm{~m}$ \\
$\rho_{\mathrm{s}}$ & Solid volumetric weight & 22000 & $\mathrm{~Pa} / \mathrm{m}$ \\
$\rho_{\mathrm{f}}$ & Fluid volumetric weight & 21000 & $\mathrm{~Pa} / \mathrm{m}$ \\
$E$ & Plate elasticity modulus & 8.3 & $\mathrm{GPa}$ \\
$L$ & Structure initial length & 2.5 & $\mathrm{~km}$ \\
\hline
\end{tabular}

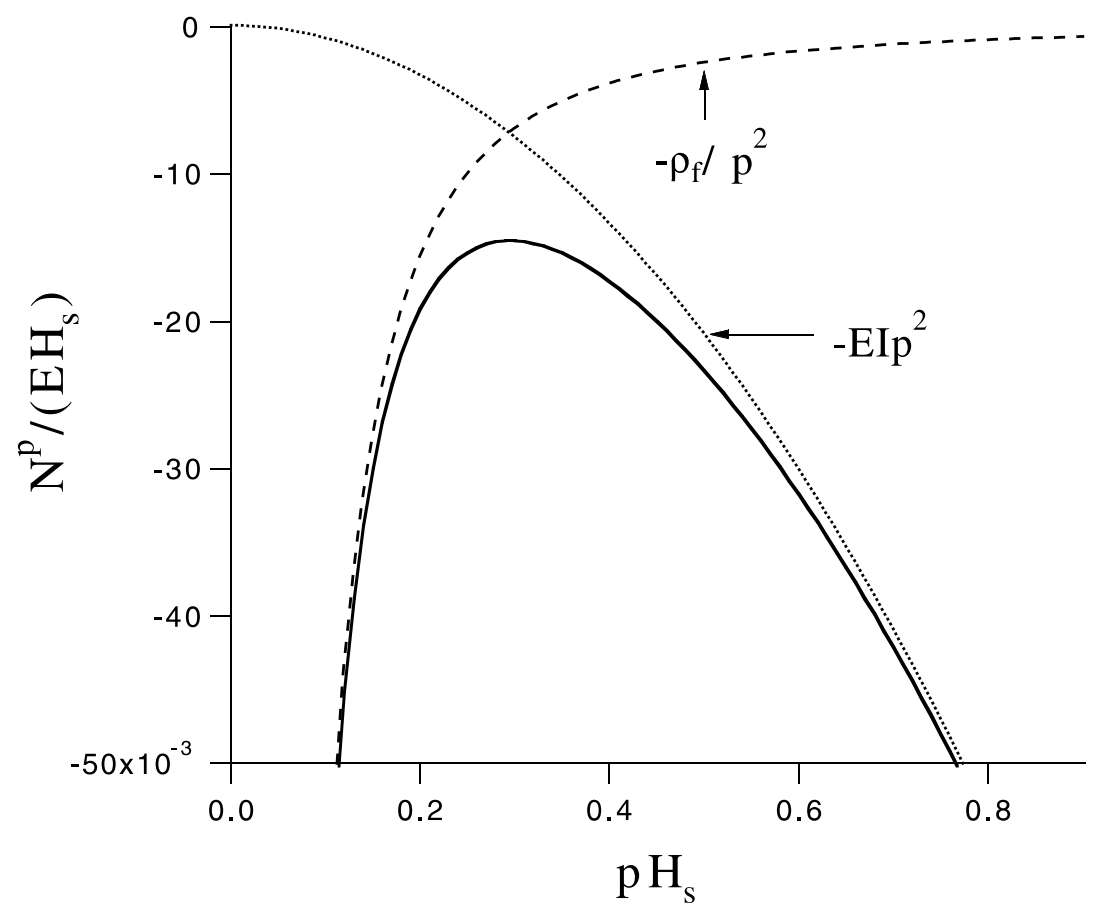

Fig. 2. The normalized critical load on the elastic plate, solid curve, as a function of the dimensionless wavenumber. This load results from two contributions, the first due to the plate bending stiffness (dotted curve) and the second due to gravity (dashed curve).

(dashed curve). The resulting critical load is the sum of these two contributions and corresponds to the solid curve in Fig. 2. It has a maximum characterized by

$$
N_{M}^{\mathrm{p}}=-2\left(E I \rho_{\mathrm{f}}\right)^{1 / 2}, \quad p_{M}=\left(\frac{\rho_{\mathrm{f}}}{E I}\right)^{1 / 4} .
$$

These results are obtained from (27) with the remark that the dimensionless number $\varepsilon \equiv \rho_{\mathrm{f}} H_{\mathrm{s}} / E$ is small compared to one. The length $L_{M}=\pi / p_{M}$ for which the compressive force magnitude is minimum is here $2661 \mathrm{~m}$. This value is surprisingly close to the 2500 estimated by Cobbold and Szatmari (1991), since the rheology in the sedimentary basin cannot be elastic. Indeed, the compressive stress for buckling $N_{M}^{\mathrm{p}} / H_{\mathrm{s}}$ is $120 \mathrm{MPa}$ and exceeds any sedimentary rock yield strength. The stress found with a deformation theory of plasticity is close to $40 \mathrm{MPa}$ (Triantafyllidis and Leroy, 1997). Despite this apparent discrepancy between stress at buckling and rock strength, and for the motivations outlined in Section 1, we continue the analysis 
of the bifurcation equilibrium path. Of interest is to establish whether buckling proceeds with an increase or a decrease in the loading parameter $\Delta$ as well as in the magnitude of the force applied on the boundary.

This question requires to determine the sign of the scalar $\Delta_{2}$ computed in the previous section. Its final expression is obtained by combining the results of (39), (42), (43) and (45) in the definition (33). This expression is certainly too complex to determine its sign. It should be however stressed that an inspection of every contribution permits to conclude that the vertical stress gradient $k$ has no influence on the initial postbifurcation. To conclude on the sign of $\Delta_{2}$, it is proposed to select the length $L_{M}$ and to approximate the expression of $\Delta_{2}$ for small $\varepsilon^{1 / 2}$ compared to one. In that instance one finds

$$
\frac{\Delta_{2}}{L_{M}}=\frac{1}{H_{\mathrm{s}}^{2}}(3 \varepsilon)^{1 / 2},
$$

and concludes that $\Delta_{2}$ is positive and thus that the bifurcated path is followed with an increase in the loading parameter $\Delta$. Knowing the sign of this scalar, we can now determine whether the fold development requires an increase in the magnitude of the total compressive force acting on the structure. For that purpose, the same asymptotic limit for small $\varepsilon$ is used to find out that the two terms yet unknown in (49), $\stackrel{2}{\omega}$ and $p v$, are of order $\varepsilon$ and $\varepsilon^{1 / 2}$, respectively. Consequently, keeping the leading term in $\varepsilon$ only, the asymptotic development (49) becomes

$$
P=P_{\mathrm{c}}-\frac{1}{2} \rho_{\mathrm{f}} \xi^{2}+\mathcal{O}\left(\xi^{3}\right)
$$

The leading term is negative and thus the compressive force magnitude decreases with the bifurcation amplitude.

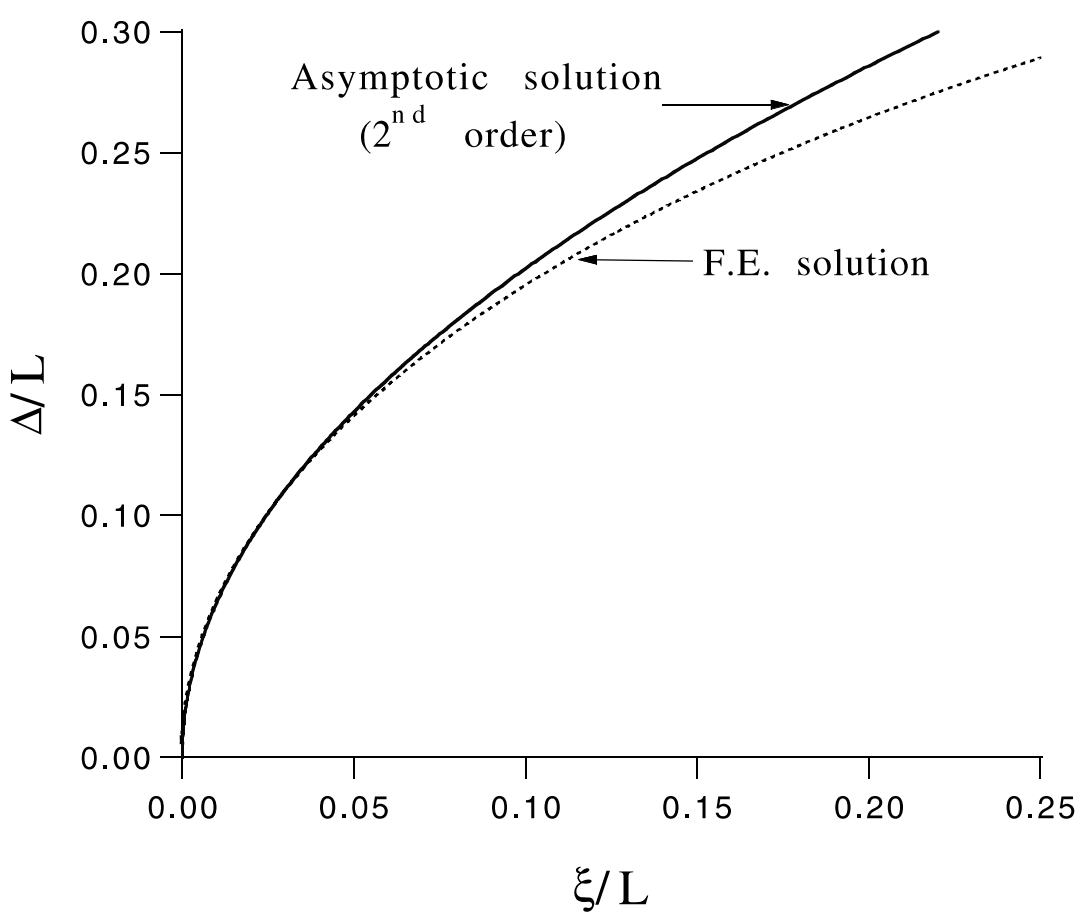

Fig. 3. The loading parameter $\Delta$ (minus the plate end displacement) as a function of the bifurcation amplitude $\xi$, according to the second-order asymptotic development (solid curve) and the finite-element solution (dotted curve). 


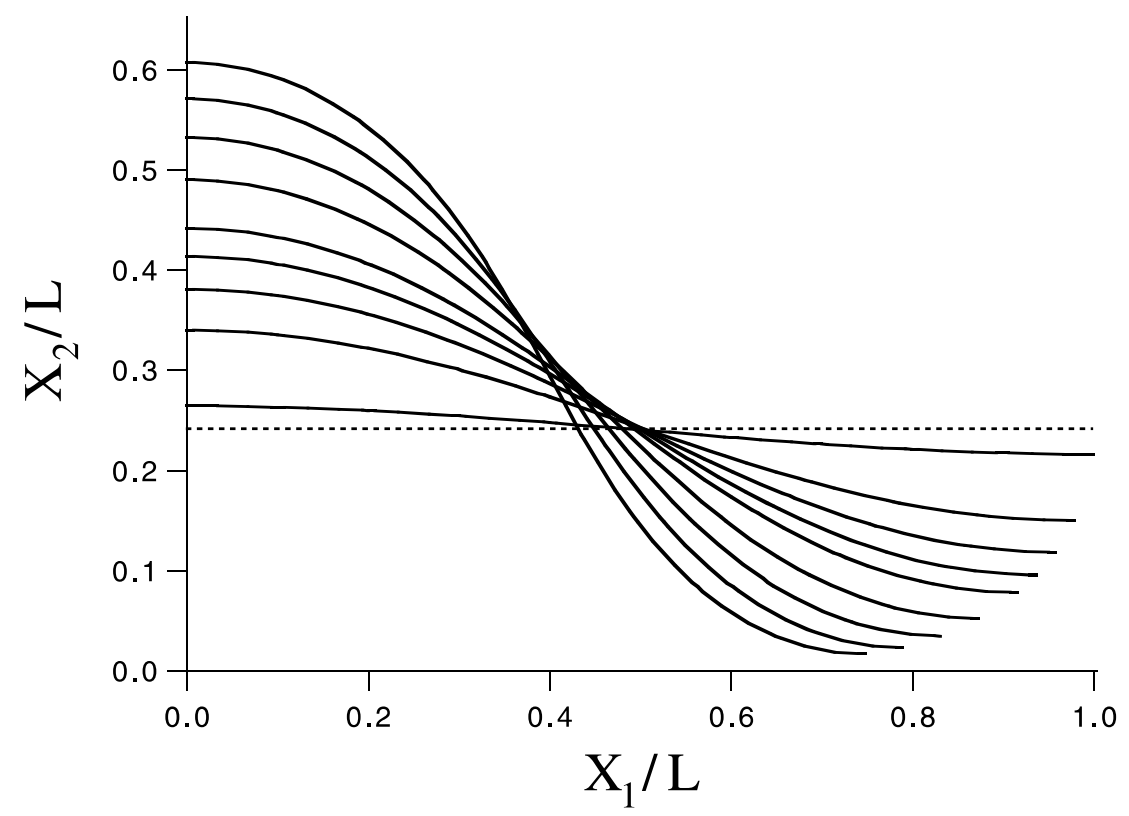

Fig. 4. The shape of the buckled plate in the post-bifurcation regime. Coordinates are normalized by the plate length. The dotted line defines the initial position of the plate.

To validate the results discussed above, the exact second-order asymptotic development is compared to the finite-element solution to the buckling problem. The finite-element approximation is presented in Appendix B. The opposite to the end displacement of the plate which is $\Delta$ is plotted as a function of the fold amplitude in Fig. 3. The dashed curve obtained with the finite-element method confirms that the scalar $\Delta_{2}$ is positive. The second-order asymptotic development, solid curve, is close to the finite-element solution and thus remains valid even for values of the normalized fold amplitude as large as 0.2. The shape of the fold, from which this amplitude is computed, is presented in Fig. 4 for different values of the end displacement at the boundary. The relevance of the post-bifurcation solutions to the geological applications of interest constrains the fold amplitude to remain small compared to the structure length defining the range for the bifurcation amplitude $\xi / L$ to be $[0 ; 0.05]$. It is for that range that the asymptotic development proposed in this paper takes its full validity.

\section{Conclusion}

The equilibrium solutions for the buckling of a plate over an inviscid and buoyant fluid have been constructed as an asymptotic development in the post-bifurcation regime using the Lyapunov-SchmidtKoiter methodology.

The buckling load results from the competing influences of the bending stiffness and the gravity, the former and the latter dominating at large and small wavenumbers, respectively. The weight of the plate results in a vertical stress which is small compared to the elasticity modulus, the ratio of the two being of the order of the scalar $\varepsilon$. As a consequence, the weight of the plate has an influence on the buckling load of order $\varepsilon$ compared to one. The vertical stress gradient, a consequence of gravity, is found to have no influence on the buckling load nor on the initial development of the bifurcation. This absence of any influence 
of the vertical stress gradient on the buckling load differs from the results of Leroy and Triantafyllidis (1996). This discrepancy is explained by the methodology used in the above reference: the starting point is a stability variational form for the stratified continuum from which the asymptotic limit of a thin overburden characteristic of a plate solution is derived.

The main result concerning the post-bifurcation regime is that bifurcation occurs with a decrease in the magnitude of the total load which is necessary to obtain an overall shortening of the structure. This result is proved here for small values of the parameter $\varepsilon$. This finding has some consequences on the interpretation of the results obtained by Massin et al. (1996) who studied a similar problem geometry for an elasto-plastic overburden resting over a viscous, incompressible fluid. The buckling load has the same expression as the

one found here with the modulus of elasticity replaced by the tangent modulus, an assumption valid if unloading is unimportant at the onset of buckling. The elasto-plastic buckling load is then of course only a fraction of the Euler load. The main result of Massin et al. (1996) is that buckling ends with the complete elastic unloading of the plate, the stratified system reaching a new equilibrium state. This result was explained by the stretching of the mid-surface during fold development conducting to a relaxation of the axial compressive load. An open question is then the link between the new equilibrium states and purely elastic solutions positioned on an equilibrium branch emanating from the Euler load. The present findings indicate that the post-bifurcation elasticity solutions require a decrease in the magnitude of the compressive force which could thus become comparable to the forces generated for the elasto-plastic post-bifurcation equilibrium solutions. A quantitative assessment of this conjecture will, however, require a complete analysis of the distribution of the permanent deformation over the plate used by Massin et al. (1996), the subject of future investigations.

\section{Appendix A. The appropriate strain measure}

The selection of a strain measure has consequences on the Euler-Lagrange equations obtained by minimizing the total energy of the system. For a plate at finite strain, the Euler-Lagrange equations transformed to the current configuration have to coincide with the classical equilibrium equations in terms of moment, shear and normal forces. The Lagrangian strain obtained with the gradient of the displacement expressed in Eq. (3), classical in continuum mechanics, does not permit to achieve this goal. The objective of this appendix is to provide a concise derivation of the appropriate strain measure for some one familiar with continuum mechanics but unaware of the contribution of Antman (1968) and Triantafyllidis and Samanta (1986).

The logical step that one could be inclined to follow to obtain a strain tensor is to start from the gradient of displacement expressed in the $\left(\mathbf{e}_{1}, \mathbf{e}_{2}, \mathbf{e}_{3}\right)$ basis and found in Eq. (3) in the main text. The displacement $\operatorname{gradient} \operatorname{Grad}(\mathbf{u})$ is

$$
\begin{aligned}
\operatorname{Grad}(\mathbf{u}) & =\frac{\partial \mathbf{u}}{\partial X_{i}} \otimes \mathbf{e}_{i} \\
& =(v-Z \sin \psi)_{, 1} \mathbf{e}_{1} \otimes \mathbf{e}_{1}+(w+Z(\cos \psi-1))_{, 1} \mathbf{e}_{2} \otimes \mathbf{e}_{1}-\sin \psi \mathbf{e}_{1} \otimes \mathbf{e}_{2}+(\cos \psi-1) \mathbf{e}_{2} \otimes \mathbf{e}_{2},
\end{aligned}
$$

in which the transversal extension is disregarded $(z=Z)$. Note that in (A.1), a summation over repeated indices implies summation from 1 to 2 and the symbol $\otimes$ is the tensorial product $((\mathbf{a} \otimes \mathbf{b}) \cdot \mathbf{c}=\mathbf{a}(\mathbf{b} \cdot \mathbf{c})$ for any three vectors $\mathbf{a}, \mathbf{b}$ and $\mathbf{c})$. The gradient of the transformation $\mathbf{F}$ defined by $\mathbf{F}=\boldsymbol{\delta}+\operatorname{Grad}(\mathbf{u})$ has for polar decomposition $\mathbf{F}=\mathbf{R} \cdot \mathbf{U}$ with

$$
\begin{aligned}
& \mathbf{R}=\cos \psi\left(\mathbf{e}_{2} \otimes \mathbf{e}_{2}+\mathbf{e}_{1} \otimes \mathbf{e}_{1}\right)+\sin \psi\left(\mathbf{e}_{2} \otimes \mathbf{e}_{1}-\mathbf{e}_{1} \otimes \mathbf{e}_{2}\right)+\mathbf{e}_{3} \otimes \mathbf{e}_{3}, \\
& \mathbf{U}=(\lambda(1+Z \kappa)-1) \mathbf{e}_{1} \otimes \mathbf{e}_{1}+\boldsymbol{\delta},
\end{aligned}
$$


so that the Lagrangian strain tensor is

$$
\mathbf{e}=\frac{1}{2}\left({ }^{\mathrm{t}} \mathbf{F} \cdot \mathbf{F}-\boldsymbol{\delta}\right)=\left(\frac{1}{2}\left(\lambda^{2}-1\right)+Z \kappa \lambda^{2}+\frac{1}{2} Z^{2} \kappa^{2} \lambda^{2}\right) \mathbf{e}_{1} \otimes \mathbf{e}_{1} .
$$

The symbol $\boldsymbol{\delta}$ introduced above denotes the second-order identity tensor. The deformation tensor in (A.3) has a single component defining the longitudinal strain. It is a quadratic function of the $Z$-coordinate. With the condition $Z \kappa \ll 1$, met by slender structures, expression (A.3) simplifies to an expression of the type

$$
(\epsilon+Z \beta) \mathbf{e}_{1} \otimes \mathbf{e}_{1},
$$

where $\epsilon$ and $\beta$ are the membrane and bending strain, respectively.

One of the objectives of the rest of this appendix is to show that the choice of $\epsilon$ and $\beta$ found in (A.3) is not appropriate to obtain the standard equilibrium equations in the current configuration. For that purpose, these equations are first recalled with the help of Fig. 5 which shows an infinitesimal length $\mathrm{d} s$ of the plate in equilibrium in the current configuration. Equilibrium requires

$$
\begin{aligned}
& \frac{\mathrm{d} \mathbf{M}}{\mathrm{d} s}-\mathbf{t} \wedge \mathbf{Q}=0, \\
& \frac{\mathrm{d}}{\mathrm{d} s}(\mathbf{Q}+\mathbf{N})+\mathbf{p}_{\mathrm{s}}=0,
\end{aligned}
$$

in which $\mathbf{M}, \mathbf{Q}, \mathbf{N}$ and $\mathbf{p}_{\mathrm{s}}$ are the bending moment, the shear force, the normal force and the load per unit length in the current configuration, respectively. Every vector could be expressed in terms of its components in the $\left(\mathbf{t}, \mathbf{n}, \mathbf{e}_{3}\right)$ basis $\left(\mathbf{M}=M \mathbf{e}_{3}, \mathbf{Q}=Q \mathbf{n}, \mathbf{N}=N \mathbf{t}\right)$ so that the equations of equilibrium become in the reference configuration

$$
\begin{aligned}
& \frac{\mathrm{d} M}{\mathrm{~d} X_{1}}-Q \lambda=0, \\
& \frac{\mathrm{d}}{\mathrm{d} X_{1}}(\mathbf{Q}+\mathbf{N})+\mathbf{p}_{1}=0,
\end{aligned}
$$

in which $\mathbf{p}_{1}$ stands for the distributed load per unit length on the reference configuration $\left(\mathbf{p}_{1}=\mathbf{p}_{\mathrm{s}} \lambda\right)$.

The axial force and bending moment $N$ and $M$ are now given an interpretation based on the material response over the plate cross-section. Assume that there exists a free energy density $\varphi(e)$ (with $e=\epsilon+\beta Z$ ) such that the first and only component of the second Piola-Kirchoff stress tensor $\left(\mathbf{S}=S \mathbf{e}_{1} \otimes \mathbf{e}_{1}\right)$ is

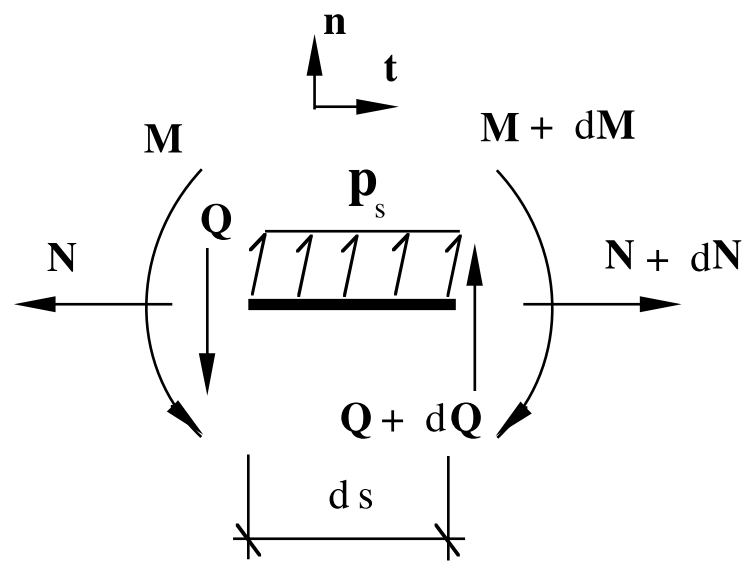

Fig. 5. The free body diagram of a segment of the plate having for infinitesimal length $\mathrm{d} s$ in the current configuration. 
$S=\partial \varphi / \partial e$. Integrating this energy density over the reference configuration cross-section results in a potential $\phi(\epsilon, \beta)$ function of the two unknown strain measures. The normal and bending components are then defined by

$$
\begin{aligned}
& N \equiv \frac{\partial \phi(\epsilon, \beta)}{\partial \epsilon}=\frac{\partial}{\partial \epsilon} \int_{-H_{\mathrm{s}} / 2}^{H_{\mathrm{s}} / 2} \varphi(e) \mathrm{d} Z=\int_{-H_{\mathrm{s}} / 2}^{H_{\mathrm{s}} / 2} S \mathrm{~d} Z \\
& M \equiv \frac{\partial \phi(\epsilon, \beta)}{\partial \beta}=\frac{\partial}{\partial \beta} \int_{-H_{\mathrm{s}} / 2}^{H_{\mathrm{s}} / 2} \varphi(e) \mathrm{d} Z=\int_{-H_{\mathrm{s}} / 2}^{H_{\mathrm{s}} / 2} S Z \mathrm{~d} Z .
\end{aligned}
$$

The stress scalar $S$ could also be interpreted as the first component of the Cauchy stress tensor in view of the transformation gradient in (A.2) and the approximation $z=Z^{2}$. The integral in (A.7) could thus be thought of as being set on the current configuration, providing the spatial interpretation of the bending and normal components which were postulated for writing the equilibrium in (A.5).

Consider now the total energy of the beam, which is the difference between the internal and external energy

$$
\mathscr{E}=\mathscr{E}^{\mathrm{i}}-\mathscr{E}^{\mathrm{e}} \quad \text { with } \mathscr{E}^{\mathrm{i}}=\int_{0}^{L} \phi(\epsilon, \beta) \mathrm{d} X_{1} \quad \text { and } \quad \mathscr{E}^{\mathrm{e}}=\int_{0}^{L} \mathbf{p}_{1} \cdot \mathbf{u d} X_{1}+\left.(-M \psi+(\mathbf{Q}+\mathbf{N}) \cdot \mathbf{u})\right|_{0} ^{L},
$$

and minimize $\mathscr{E}$ to obtain the equilibrium conditions

$$
\int_{0}^{L}\left[N \widetilde{\epsilon}+M \widetilde{\beta}-\mathbf{p}_{1} \cdot \widetilde{\mathbf{u}}+\frac{\mathrm{d}}{\mathrm{d} X_{1}}(M \widetilde{\psi}-(\mathbf{Q}+\mathbf{N}) \cdot \widetilde{\mathbf{u}})\right] \mathrm{d} X_{1}=0,
$$

in terms of the variations $\widetilde{\epsilon}$ and $\widetilde{\beta}$ in the unknown strain measures, in angle $\widetilde{\psi}$ and in displacement $\widetilde{\mathbf{u}}$. It remains now to differentiate the term in parenthesis in the integrand, to include the equilibrium equations (A.6) and to make use of the relations $\widetilde{\mathbf{u}}_{1}=\widetilde{\lambda} \mathbf{t}+\widetilde{\psi} \lambda \mathbf{n}$ to obtain

$$
\int_{0}^{L} N(\widetilde{\epsilon}-\widetilde{\lambda})+M\left(\widetilde{\beta}+\widetilde{\psi}_{, 1}\right) \mathrm{d} X_{1}=0 .
$$

This equation requires

$$
\widetilde{\epsilon}=\widetilde{\lambda} \quad \text { and } \quad \widetilde{\beta}=-\widetilde{\psi}_{, 1}=\widetilde{\kappa \lambda} \text {. }
$$

The choice for $\beta$ and $\epsilon$ obtained from the Lagrangian strain tensor in (A.3) does not permit to satisfy this criterion and thus cannot be considered for the admissible strain measure. To obtain the expected equilibrium equation, one needs to select $\beta=\kappa \lambda$ and $\epsilon=\lambda-1$. These expressions define the strain measure which enter the definition of the strain tensor $(\epsilon+\beta Z) \mathbf{e}_{1} \otimes \mathbf{e}_{1}$ denoted $\mathbf{e}$ in the main text.

\section{Appendix B. The finite-element solution}

The objective of this appendix is to construct the finite-element approximation to the solution of (14). For that purpose $\omega\left(X_{1}=0\right)$ is set to zero to select a decomposition in (10).

The displacement discretization for the variable $\mathbf{v}=(v, \omega)$ over a two-noded element of length $L_{e}$ is

$$
\mathbf{v}=\mathbf{N} \cdot \mathbf{q} \quad \text { with } \mathbf{N}=\left[\begin{array}{cccccccc}
H_{1} & H_{2} \frac{L_{e}}{2} & 0 & 0 & H_{3} & H_{4} \frac{L_{e}}{2} & 0 & 0 \\
0 & 0 & H_{1} & H_{2} \frac{L_{e}}{2} & 0 & 0 & H_{3} & H_{4} \frac{L_{e}}{2}
\end{array}\right],
$$

\footnotetext{
${ }^{2}$ To reach this conclusion, use the transformation rule $J \mathbf{F} \cdot \mathbf{S} \cdot{ }^{\mathrm{t}} \mathbf{F}$ to obtain the Cauchy stress $J S \mathbf{t} \otimes \mathbf{t}$ and approximate the Jacobian of the transformation $J$ by one, its zero order in $\kappa Z$.
} 
in which $\mathbf{q}$ is the collection of nodal variables $\left(v_{1}, V_{1}, \omega_{1}, \Omega_{1}, v_{2}, V_{2}, \omega_{2}, \Omega_{2}\right)$ where the subscript identifies the node and $V$ and $\Omega$ denote the derivatives with respect to the coordinate $X_{1}$ of $v$ and $\omega$, respectively. The array $\mathbf{N}$ in (B.1), which should not be confused with the normal to the beam in the reference configuration, is based on the four Hermite polynomials denoted $H_{\alpha},(\alpha=1, \ldots, 4)$. The first and second derivatives with respect to $X_{1}$ of the displacement are constructed similarly to (B.1)

$$
\mathbf{v}_{, 1}=\mathbf{M} \cdot \mathbf{q} \text { and } \mathbf{v}_{, 11}=\mathbf{O} \cdot \mathbf{q} .
$$

The $2 \times 8$ arrays $\mathbf{M}$ and $\mathbf{O}$ differs simply from the definition of $\mathbf{N}$ in (B.1) by the derivatives with respect to $X_{1}$. Note that in this appendix the notation introduced in the main text for tensors is extended here to matrices which are also identified by brackets []. The notation () is reserved for line vectors. The admissible strain vector $\epsilon_{\mathrm{v}}[\widetilde{v}]$ is now defined as the collection ${ }^{\mathrm{t}}\left(\epsilon_{\mathrm{v}}, \beta_{\mathrm{v}}\right)[\widetilde{v}]$ of two linear operators introduced in (15) and is written as

$$
\boldsymbol{\epsilon}_{\mathbf{v}}[\widetilde{v}]=\mathbf{B} \cdot \widetilde{\mathbf{q}} \quad \text { with } \mathbf{B}=\left[\begin{array}{c}
\cos (\psi) M_{1 i}+\sin (\psi) M_{2 i} \\
\frac{v, 11}{\lambda^{2}} M_{2 i}+\frac{\sin (\psi)}{\lambda} O_{1 i}-\frac{\cos (\psi)}{\lambda} O_{2 i}-\frac{\omega_{21}}{\lambda^{2}} M_{1 i}-\frac{2 \beta}{\lambda} B_{1 i}
\end{array}\right],
$$

in terms of the $2 \times 8$ matrix $\mathbf{B}$ defined for its two lines and for the column $i$ in terms of the components of $\mathbf{M}$ and $\mathbf{O}$ found in (B.2).

The element contribution to the equilibrium equations is ${ }^{\mathrm{t}} \widetilde{\mathbf{q}} \cdot \mathbf{F}$ with

$$
\begin{aligned}
& \mathbf{F}=\int_{0}^{L_{e}}{ }^{\mathrm{t}} \mathbf{B} \cdot \boldsymbol{\sigma}+a \mathbf{N}_{2}+b \mathbf{M}_{1} \mathrm{~d} X_{1}, \\
& a=\rho_{\mathrm{s}} H_{\mathrm{s}}\left(1-\frac{L}{l}\left(1+v_{, 1}\right)\right)+\rho_{\mathrm{f}}\left(\omega+W-H_{\mathrm{f}} \frac{L}{l}\right)\left(1+v_{, 1}\right), \\
& b=\omega\left(-\rho_{\mathrm{s}} H_{\mathrm{s}} \frac{L}{l}+\rho_{\mathrm{f}}\left(\frac{\omega}{2}+W-H_{\mathrm{f}} \frac{L}{l}\right)\right),
\end{aligned}
$$

in which the column vector $\boldsymbol{\sigma}, \mathbf{N}_{2}$ and $\mathbf{M}_{1}$ are the pair ${ }^{\mathrm{t}}(N, M)$ and the column vectors composed of the second and first line of the arrays $\mathbf{N}$ and $\mathbf{M}$, respectively. This equation (B.4) is the finite-element approximation of (14) with the only difference that the zero-datum for $W$ is set at the basis of the structure. The linearization of (B.4) for a Newton-Raphson equilibrium search provides the following element contribution to the global set of equations:

$$
\begin{aligned}
& { }^{\mathrm{t}} \widetilde{\mathbf{q}} \cdot\left(\mathbf{K}-\frac{\rho_{\mathrm{f}}}{l} \mathbf{Q} \otimes \mathbf{Q}\right) \cdot \widehat{\mathbf{q}}=-{ }^{\mathrm{t}} \widetilde{\mathbf{q}} \cdot \mathbf{F} \quad \text { with } \widetilde{W}=-\frac{1}{l} \mathbf{Q} \cdot \widetilde{\mathbf{q}} \quad \text { and } \quad \mathbf{Q}=\int_{0}^{L_{e}}\left(1+v_{, 1}\right) \mathbf{N}_{2}+\omega \mathbf{M}_{1} \mathrm{~d} X_{1}, \\
& \mathbf{K}=\int_{0}^{L_{e}}\left\{{ }^{\mathrm{t}} \mathbf{B} \cdot \mathbf{D} \cdot \mathbf{B}+g_{1}{ }^{\mathrm{t}} \mathbf{M} \cdot \mathbf{M}+g_{2}{ }^{\mathrm{t}} \mathbf{P} \cdot \mathbf{R} \cdot \mathbf{P}+g_{3}\left(\mathbf{N}_{2} \otimes \mathbf{M}_{1}+\mathbf{M}_{1} \otimes \mathbf{N}_{2}\right)+g_{4} \mathbf{N}_{2} \otimes \mathbf{N}_{2}\right\} \mathrm{d} X_{1}, \\
& \mathbf{D}=\left[\begin{array}{cc}
\frac{\left(E H_{\mathrm{s}}-N_{\mathrm{c}}^{\mathrm{p}}\right)}{\lambda} & -\frac{2 M}{\lambda} \\
-\frac{2 M}{\lambda} & E I
\end{array}\right], \quad \mathbf{P}=\left[\begin{array}{c}
M_{1 i} \\
O_{1 i} \\
M_{2 i} \\
O_{2 i}
\end{array}\right], \quad \mathbf{R}=\left[\begin{array}{cccc}
0 & 0 & 0 & 1 \\
0 & 0 & -1 & 0 \\
0 & -1 & 0 & 0 \\
1 & 0 & 0 & 0
\end{array}\right], \\
& g_{1}=\left(N-\frac{2 \beta M}{\lambda}\right) \lambda^{-1}, \quad g_{2}=-\frac{M}{\lambda^{2}}, \quad g_{3}=\rho_{\mathrm{f}}\left(\omega+W-\frac{L}{l} H_{\mathrm{f}}\right)-\rho_{\mathrm{s}} H_{\mathrm{s}} \frac{L}{l}, \quad g_{4}=\rho_{\mathrm{f}}\left(1+v_{, 1}\right) .
\end{aligned}
$$

The $\mathbf{Q} \otimes \mathbf{Q}$ contribution in (B.5) to the global stiffness array ruins its band structure and a special solution procedure is now proposed. Using the same notation at the global level as at the element level, it consists of decomposing the global solution vector $\widehat{\mathbf{q}}$ into two parts $\widehat{\mathbf{q}}_{1}+\alpha \widehat{\mathbf{q}}_{2}$ with the scalar $\alpha$ set to $-\rho_{\mathrm{f}} \widehat{W}$. The vectors $\widehat{\mathbf{q}}_{1}, \widehat{\mathbf{q}}_{2}$ and the increment $\widehat{W}$ are then found by solving 


$$
\mathbf{K} \cdot \widehat{\mathbf{q}}_{1}=-\mathbf{F}, \quad \mathbf{K} \cdot \widehat{\mathbf{q}}_{2}=\mathbf{Q}, \quad \widehat{W}=\frac{-\mathbf{Q} \cdot \widehat{\mathbf{q}}_{1}}{l-\rho_{\mathrm{f}} \mathbf{Q} \cdot \widehat{\mathbf{q}}_{2}}
$$

which makes use of the band-structure of the global stiffness array $\mathbf{K}$.

\section{References}

Antman, S., 1968. General solutions for plane extensible elastic having nonlinear stress-strain laws. Quart. Appl. Math. $26,35-47$. Budiansky, B., 1974. Theory of buckling and postbuckling behavior of elastic structures. In: Yih, C.H. (Ed.), Advances in Applied Mechanics. Academic Press, New, pp. 1-65.

Cobbold, P.R., Szatmari, P., 1991. Radial gravitational gliding on passive margins. Tectonophysics 188, $249-289$.

Guiton, M.L.E., Leroy, Y.M., Sassi, W., 2001. Activation of diffuse discontinuities and folding of sedimentary layers. Submitted for publication.

Iooss, G., Joseph, D.D., 1980. Elementary stability and bifurcation theory. Springer, Berlin.

Leroy, Y.M., Triantafyllidis, N., 1996. Stability of a frictional, cohesive layer on a substratum: variational formulation and asymptotic solution. J. Geophys. Res. 101, 17,795-17,811.

Massin, P., Triantafyllidis, N., Leroy, Y.M., 1996. Stability of a density-stratified two-layer system. C.R. Acad. Sci., Sér. IIa, 322, 407413.

Martinod, J., Davy, P., 1992. Periodic instabilities during compression or extension of the lithosphere. 1-Deformation modes from an analytical perturbation method. J. Geophys. Res. B 97 (2), 1999-2014.

McAdoo, D.C., Sandwell, D.T., 1985. Folding of the oceanic lithosphere. J. Geophys. Res. 90, 8563-8569.

Potier-Ferry, M., 1978. Bifurcation et stabilité pour des systèmes dérivant d'un potentiel. J. de Mécanique 17, 579-608.

Potier-Ferry, M., 1979. Perturbed bifurcation theory. J. Differ. Equations 33, 112-146.

Ramberg, H.R., Stephansson, O., 1964. Compression of floating elastic and viscous plates affected by gravity, a basis for discussing crustal buckling. Tectonophysics 1, 101-120.

Triantafyllidis, N., Samanta, S.K., 1986. Bending effects on flow localization in metallic sheets. Proc. Roy. Soc. Lond. A 406, $205-226$.

Triantafyllidis, N., Leroy, Y.M., 1997. Stability of a frictional, cohesive layer on a substratum: validity of asymptotic solution and influence of material properties. J. Geophys. Res. B 102 (9), 20551-20570.

Triantafyllidis, N., Peek, R., 1992. On stability and the worst imperfection shape in solids with nearly simultaneous eigenmodes. Int. J. Solids Struct. 18, 2281-2299.

Wallace, M.H., Melosh, H.J., 1994. Buckling of a pervasively faulted lithosphere. Pure Appl. Geophys. 142, $239-261$. 